\title{
Rotation of axes for anisotropic metal in FEM simulations
}

\author{
L. Duchêne ${ }^{\mathrm{a}}$, T. Lelotte ${ }^{\mathrm{b}}$, P. Flores ${ }^{\mathrm{d}}$, S. Bouvier ${ }^{\mathrm{c}}$, A.-M. Habraken ${ }^{\mathrm{b}}$ \\ ${ }^{a}$ Cobo Dept., Royal Military Academy, av. Renaissance 30, 1000 Brussels, Belgium \\ ${ }^{\mathrm{b}}$ ArGEnCo Dept., University of Liège, Chemin des Chevreuils 1, 4000 Liège, Belgium \\ ${ }^{\mathrm{c}}$ LPMTM-CNRS UPR9001, University Paris 13, 99 av. J.B. Clément, 93200 Villetaneuse, France \\ ${ }^{\mathrm{d}}$ DIM, Universidad de Concepcion, Casilla 160-C - Correo 3, Concepcion, Chile
}

\begin{abstract}
For the FE simulations relying on elasto-plastic models based on anisotropic yield locus description, it is important for the simulation accuracy to follow a Cartesian reference frame, where the yield locus is expressed. The classical formulations like the Hill 1948 model keep a constant shape of the yield locus when other texture based yield loci regularly update their shape. However in all these cases, the rotation of the Cartesian reference frame must be known. For simple shear tests performed on steel sheets, experimental displacements provide the actual updated position of initial orthogonal grids. The initial and final texture measurements give information on the average crystals rotation. For Hill constitutive law and texture based models, this paper compares the experimental results with different ways to follow the Cartesian reference frame: the co-rotational method, an original method based on the constant symmetric local velocity gradient and the Mandel spin computed by four different methods.
\end{abstract}

Keywords: B. Anisotropic material; B. Constitutive behaviour; B. Crystal plasticity; B. Finite strain; C. Finite elements

\section{INTRODUCTION}

Today, the material forming processes are often studied by finite element (FE) simulations. These numerical analyses help the engineers to better understand the material answer during strong plastic deformations or in complex strain paths. In deep drawing process for instance, it predicts the punch force, the final geometry of the work-piece, its residual stress and strain fields (Li et al., 1995; Duchêne, 2003; Habraken and Duchêne, 2004; Yoon et al., 2004; Yoon et al., 2006). The next step already applied in some industrial sectors is the optimization of the tool geometry or the internal pressure and die displacement laws like in the hydro-forming process (Johnson et al., 2004).

In FE simulations describing large plastic strains, three frames coexist (Fig. 1): (i) the global frame $\{X, Y, Z\}$ where the mesh is defined, which is Cartesian and fixed during the whole simulation, (ii) the actual material frame $\left\{x^{\prime}, y^{\prime}, z^{\prime}\right\}$, initially three orthogonal lines drawn on the material which become curve and non-Cartesian as the material deforms, this frame is typically unknown in FE simulations and finally (iii) the Cartesian reference frame $\{\mathrm{RD}, \mathrm{TD}, \mathrm{ND}\}$ called hereafter the local axes which remain orthogonal, where material anisotropy coefficients describe the material and which tries to propose an average position of the material frame. In the present work, the Cartesian reference frame is initially chosen aligned with the rolling direction RD, the transverse direction TD and the normal direction ND of the rolled sheet. The three axes Z, z' and ND are coaxial.

During plastic deformation of solids, the existence (and the continuous evolution) of symmetry axes of the material throughout the deformation is not guaranteed. It depends on the initial symmetry of the material and, above all, on the symmetries of the forming process. If the (initial) symmetry axes of the material and the forming process are misoriented (consider e.g. a tensile test at $45^{\circ}$ from $\mathrm{RD}$ of a metal sheet), a 'jump' of the symmetry axes of the material can be observed. In spite of these special cases, the definition of orthogonal local axes continuously evolving during plastic deformation is in general very useful in order to express the material's constitutive law.

The FE simulation of a simple shear test is one of the most critical cases (Dafalias, 1983), while it presents two problems: strong rotation and distortion of the material frame. The present paper studies the effect of different 
choices defining the local axes position on the final description of the material anisotropy. To identify the best approach, FE simulation results of simple shear tests are compared with experimental observations (the displacement field provided by optical extensometer and the texture measurements) for one steel grade. Three methods to compute FE local axes evolution are tested: (i) an assumption of Jaumann type based on the skewsymmetric part of the velocity gradient and called "Constant Symmetric Local Velocity Gradient (CSLVG)" with or without crystallographic texture update (i.e. kinematics based approach), (ii) the co-rotational frame directly computed inside each finite element from the nodal displacement fields (i.e. purely geometrical approach), (iii) the Mandel spin estimated as an average rotation of a set of crystals representative of the crystallographic texture (i.e. more physical approach).

Fig. 1: Axes definition in simple shear test. $\{X, Y, Z\}$ indicates the global frame, $\{R D, T D, N D\}$ indicates the local frame, $\left\{x^{\prime}, y^{\prime}, z^{\prime}\right\}$ indicates the material frame: $(a)$ initial configuration and $(b)$ updated configuration.

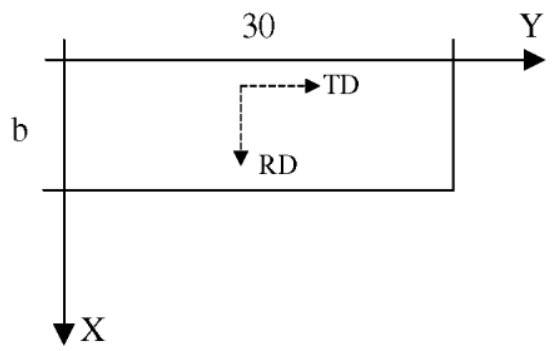

b

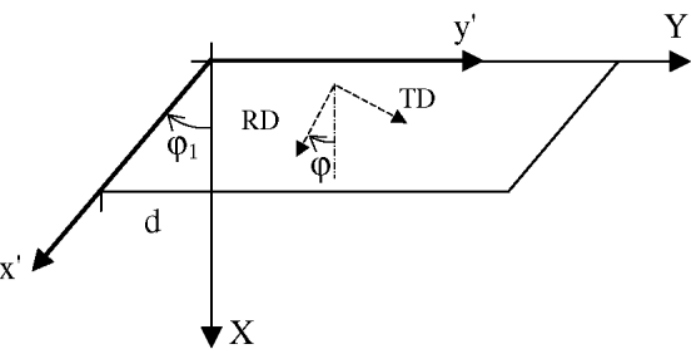

The validity of these three rotation methods has previously been checked thanks to different simple tests (e.g. tensile tests along RD and at $45^{\circ}$ from RD).

In the industrial simulations, analytical yield loci like the classical (Hill, 1948) one for steel grades or the (Barlat et al., 1991) one for aluminium alloys are often used (see also Kim et al., 2007). They provide reasonable accuracy and CPU time. For such models, the initial material anisotropy coefficients are defined in the local frame whose initial orientation is easily identified in cold rolled metal sheets by the rolling direction (RD), the transverse direction (TD) and the normal direction (ND) of the sheet. In classical phenomenological models (Hill, 1948; Barlat et al., 1991; Yoon et al., 2006), the yield locus shape and by consequence the material anisotropy coefficients are assumed constant, so they must be expressed in a correctly oriented frame. The yield locus size is defined by the chosen isotropic hardening model and its position by the chosen kinematic hardening model. Other teams use micro-macro approaches, where the texture evolution is known and used from time to time, to update the yield locus shape (Van Houtte and Van Bael, 2004). Between the updating steps of the yield locus after a given strain interval (typically after $20 \%$ of plastic deformation in Peeters et al. (2001)), they rely on a constant yield locus shape that must again be expressed in a specific local frame. In both cases, the way the local axes rotations are computed can increase the simulation accuracy. When fully micro-macro computations (i.e. the macroscopic stress tensor is the average of the microscopic stress tensors computed on a collection of representative crystals whose orientation is computed at each FE increment) are applied as in Dawson et al. (2005) or Delannay et al. (2006), no local axes are required.

The last two decades, the local axes definition has already been given a lot of attention. For instance previous analyses were published by Mandel (1982), Hughes (1983), Dafalias (1983, 1985, 1998), Van Der Giessen (1991), Wu (2007). The specific case of simple shear was particularly studied in Bacroix et al. (1994) and Peeters et al. (2001).

In nonlinear FEM package, hypo-elastic law can be used in order to link the objective derivative $\underset{\sigma}{\sigma}$ of the Cauchy stress tensor $\sigma$ to the strain rate D (the symmetric part of the velocity gradient L) by

$$
\stackrel{\nabla}{\boldsymbol{\sigma}}=\dot{\boldsymbol{\sigma}}-\boldsymbol{\Omega} \boldsymbol{\sigma}+\boldsymbol{\sigma} \boldsymbol{\Omega}=\mathbf{C}: \mathbf{D}
$$

where $\mathrm{C}$ is the tangent modulus, $\dot{\boldsymbol{\sigma}}$ is the derivative of the stress tensor and $\Omega$ is a second order skew-symmetric spin. All these tensors are assumed to be expressed in the global reference frame (see Fig. 1). The right hand side of Eq. (1) accounts for the actual rheological law, where the terms $\Omega \sigma-\sigma \Omega$ define the stress variation expressed 
in the global reference frame when a large rotation of the continuum media occurs. When the spin tensor $\Omega$ is the skew-symmetric part $\mathrm{W}$ of the velocity gradient $\mathrm{L}$, the Jaumann derivative is recovered. In this case, $\mathrm{W}$ is also called the rigid body spin. However looking at polycrystalline materials where plastic deformations take place by dislocation movements along slip planes in slip directions, one can find that a more physical spin can be computed. If the slip system $s$ is determined by the unit vector $b^{s}$ showing the slip direction and the unit vector $n^{s}$ defining the normal on the slip plane, any microscopic velocity gradient $\mathrm{L}^{\text {micro }}$ can be expressed as the results of slip rates $\dot{\gamma}^{s}$ on different slip systems and a rate of crystal lattice rotation $\omega^{\text {lattice }}$ as shown by Eq. (2):

$$
L_{i j}^{\text {micro }}=\sum_{s} b_{i}^{s} n_{j}^{s} \dot{\gamma}^{s}+\omega_{i j}^{\text {lattice }}
$$

The crystal rigid body spin tensor $\mathrm{w}$ can now be decomposed as a plastic spin tensor $\omega^{\text {plastic }}$ related to the internal crystal slips and the crystal lattice rotation $\omega^{\text {lattice }}$ :

$$
w_{i j}=\frac{1}{2}\left(L_{i j}^{\text {micro }}-L_{j i}^{\text {micro }}\right)=\frac{1}{2} \sum_{s}\left(b_{i}^{s} n_{j}^{s}-b_{j}^{s} n_{i}^{s}\right) \dot{\gamma}^{s}+\omega_{i j}^{\text {lattice }}=\omega_{i j}^{\text {plastic }}+\omega_{i j}^{\text {lattice }} \text {. }
$$

These three different rotations or spins: $w, \omega^{\text {plastic }}, \omega^{\text {lattice }}$ are represented in Fig. 2 for the simple case of one activated slip system.

Fig. 2: Schematic representation of the different crystal spins.
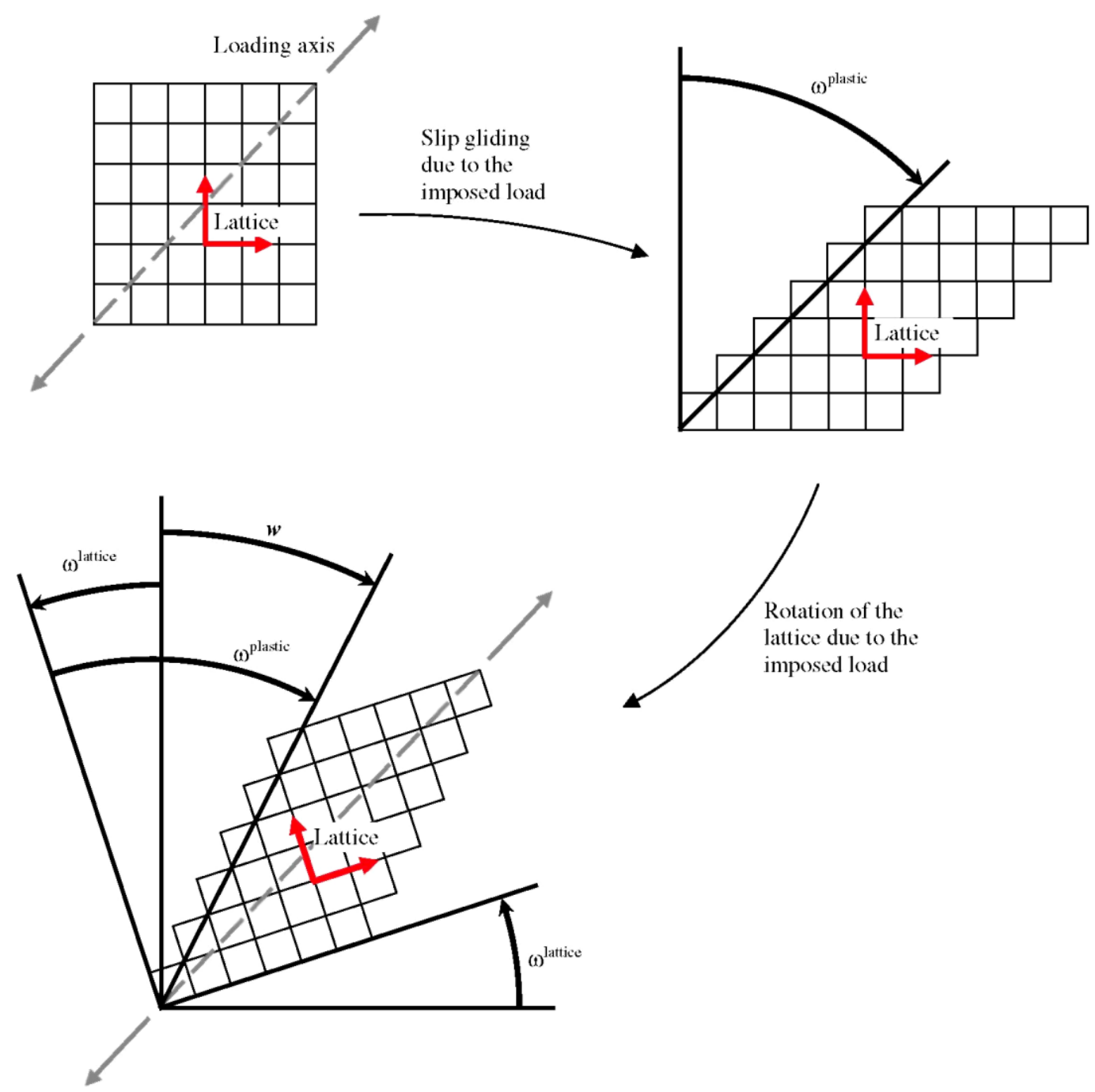
The polycrystal behaviour can be predicted using a micro-mechanical model as the well-known Full Constraint Taylor's model where an assumption of equality between the microscopic plastic velocity gradient of each grain and the macroscopic plastic velocity gradient is imposed. In this case, the macroscopic rigid body spin $\mathrm{W}$ can be decomposed in two terms, $\mathrm{W}^{\text {plastic }}$ and $\mathrm{W}^{\text {lattice }}$ seen as the average of what happens on the crystal level (Dafalias, 1985).

$$
\mathbf{W}=\mathbf{W}^{\text {plastic }}+\mathbf{W}^{\text {lattice }}
$$

Mandel (1982) already proposed that the macroscopic local frame should be linked to the material microstructure where the crystallographic lattice defines reference directions. The so-called Mandel spin here written $\mathrm{W}^{\text {lattice }}$; is nothing else than the average of the rotation rate of the crystal frames. Following his idea, the effect of large deformation should be described by this Mandel spin and not the rigid body rotation as used in the Jaumann derivative. However in phenomenological macroscopic models, it is quite hard to find constitutive evolution laws for both terms of Eq. (4), even if Van Der Giessen (1991) proposed some theory based on the thermodynamic and micromechanic approach.

For simple shear test, Bacroix et al. (1994) investigate the evolution of a privileged frame using the Mandel spin, the Taylor's model and the initial texture. As previously mentioned by Toth et al. (1990), the authors find that the average spin has only one non-zero component which corresponds to a rotation around the normal direction. Moreover, they observe that the loss of orthotropy seems to be minimized with the Mandel spin. Peeters et al. (2001) used a constant Taylor-Bishop-Hill model identified from an initial texture coupled with three different approaches to define the local axes position: (i) the macroscopic Jaumann approach or rigid body spin W, (ii) the use of an accurate Mandel spin $\mathrm{W}^{\text {lattice }}$ taking into account the texture evolution computed by the Full Constraints Taylor model (Van Houtte and Rabet, 1997) and (iii) a simplified Mandel spin $\mathrm{W}^{\text {rough lattice }}$ calculated without texture updating or with texture updating after successive strain intervals using the same micro-mechanical model. The authors show that the results are sensitive to the initial texture. When the latter is far from the stable texture for the corresponding imposed strain mode, the strong texture evolution during the material straining shows that the true Mandel spin $\mathrm{W}^{\text {lattice }}$ provides a better answer than the rigid body spin W. Moreover, the authors show that simplified Mandel spin $\mathrm{W}^{\text {rough lattice }}$ does not improve the results when the texture is not updated. However, in case of stable initial texture for the imposed strain mode, the Mandel spin $\mathrm{W}^{\text {lattice }}$ is still quite far from the rigid body spin $\mathrm{W}$ but this time is well approximated by the simplified Mandel spin $\mathrm{W}^{\text {rough lattice }}$ even when the texture is not updated. Finally, Peeters et al. (2001) show that when the texture is updated after a given strain interval, the results given by $\mathrm{W}^{\text {rough lattice }}$ approximate quite well the ones obtained by $\mathrm{W}^{\text {lattice }}$ in all cases (stable and unstable texture). Yoon et al. (2005) analysed the simple shear test using FE computation with two constitutive models (phenomenological model and Taylor-Bishop-Hill model). The investigations were carried out on two highly anisotropic materials. The authors show that crystal plasticity which accounts for texture evolution, leads to a real anisotropic hardening effect. However, the authors did not discuss the effect of the chosen co-rotational coordinate systems on their results.

The present paper will focus more on macroscopic FEM solutions as it will compare the macroscopic CSLVG approach, with (i) the co-rotational classical approach, and with (ii) the $\mathrm{W}^{\text {lattice }}$ approach coupled with a constant or updated yield locus (i.e. with or without crystallographic texture update). As the computation of $\mathrm{W}^{\text {lattice }}$ is a key issue in the practical use of this approach, four methods are proposed for its estimation. For an IF mild steel, experimental validations based on initial and final texture measurements as well as on displacement fields are provided. In Section 2 the experimental device used to perform simple shear tests is described. The method used for the measurement of displacement fields is briefly recalled. The material used in this work and the initial texture measurement are presented. Sections 3 and 4 describe the FE package with the different elements and methods used to predict the local axes rotation. Section 5 contains the FE results using different yield loci (Hill, 1948 and the so-called Minty yield surface (Habraken and Duchêne, 2004) computed using the initial texture). In this work, for completeness an updated yield locus (namely Evol yield surface) based on an updated texture is also considered. The adopted yield surface descriptions are coupled with co-rotational approach, CSLVG method and Mandel spin estimation to follow the local axes evolution and compare them to experimental results. Finally, Section 6 gives some concluding remarks and perspectives of the proposed work. 


\section{EXPERIMENTAL PROCEDURE AND MATERIAL CHARACTERISATION}

\subsection{Testing equipment}

An original bi-axial machine was developed using hydraulic grips (Flores, 2005; Flores et al., 2005) (Fig. 3). The latter are moved under controlled displacement and force, simultaneously or independently. Therefore, simple shear test or plane strain test can be performed at the same time or separately. The acquisition system records the loading force, the grip displacement and the strain field using a full-field optical measurement technique based on ARAMIS (Gom, 2001). This non-contact measurement system gives the possibility to have the strain field along the gauge area of the deformed specimen. Therefore, several experimental error sources are controlled such as the effect of the free-end specimen on the homogeneity of the strain field, the possible slip of the sample under the grip area.

The gauge area of the simple shear sample is a rectangular shape $30 \mathrm{~mm}$ long and $3 \mathrm{~mm}$ wide (= b in Fig. 1). The geometric conditions to reach a large homogeneous strain field are respected (Bouvier et al., 2006), as confirmed latter by the optical strain measurement (Fig. 4). The different frames used in the present work are shown in Fig. 1. The imposed amount of shear strain $\gamma=2 \varepsilon_{12}=\tan \varphi_{1}=d / b$ is achieved through the value of $\mathrm{d}$. In the present work, the sample was deformed up to $\gamma=70 \%$, which corresponds to an angle $\varphi_{1} \approx 35^{\circ}$. Experimentally, the optical system has measured in a small element at the centre of the sample, a rotation $\varphi_{I}=$ $37.2^{\circ}$ for this required displacement confirming some small inaccuracy in experiment (machine stiffness, slip in the grips, testing machine control, etc.).

Fig. 3: Bi-axial testing machine designed at the ArGEnCo Laboratory (Flores, 2005; Flores et al., 2005).

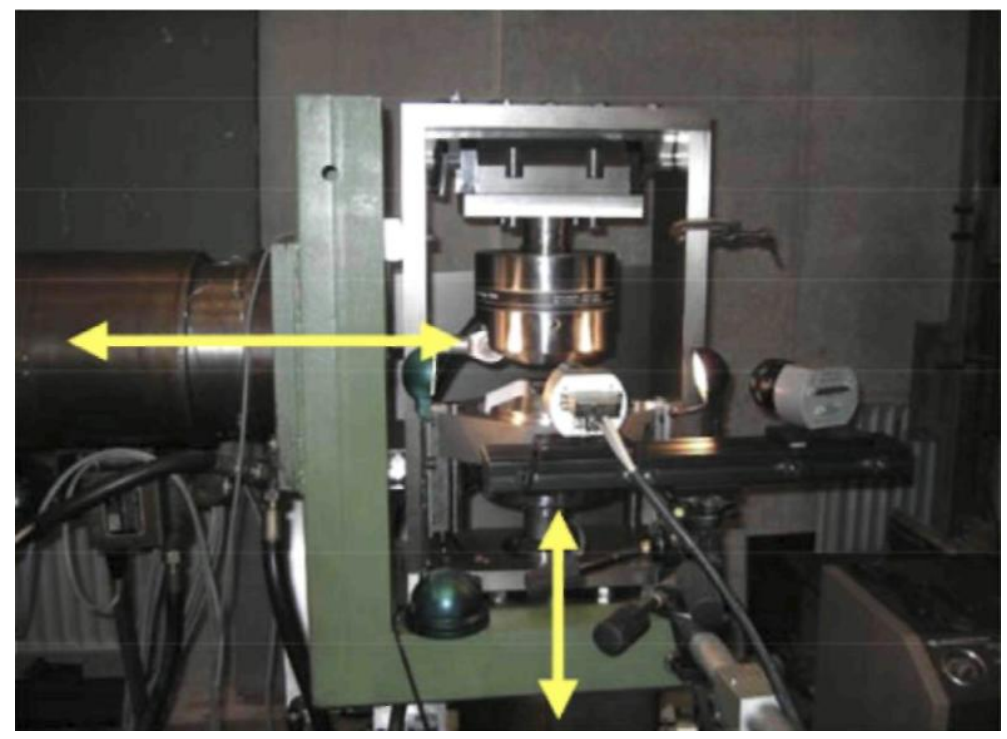

Fig. 4: FeP06t, IF mild steel: amount of shear strain field computed by ARAMIS for an imposed displacement $d=2.1 \mathrm{~mm}$, leading to $\gamma=0.7$.

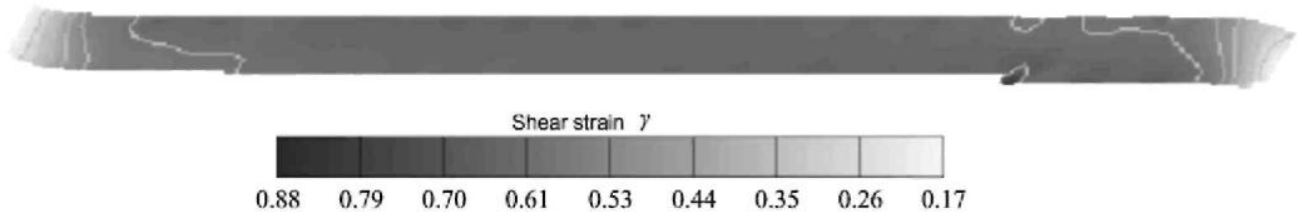


Fig. 5: As-received FeP06t mild steel, (110)- and (100)-experimental pole figures.
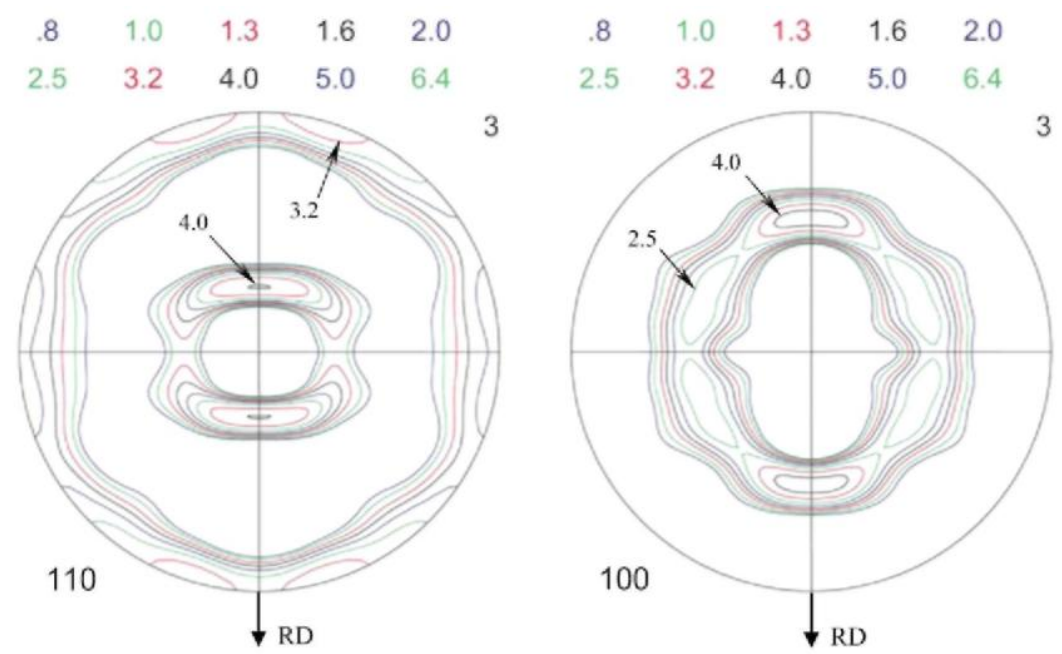

\subsection{Texture measurement}

As it is required by the models used in the present study, the texture measurements for the as-received and deformed material were achieved by standard X-rays diffraction techniques at mid-thickness of the sheet. Four incomplete pole figures $\{(110),(200),(211)$ and (301) $\}$ were measured. For simplicity, only the (110) and (100) recalculated pole figures are presented. The initial texture for the non-deformed FeP06t IF mild steel sheet of 0.7 $\mathrm{mm}$ thickness is depicted in Fig. 5 where the rolling direction goes downward as indicated. The threedimensional ODF $f(\mathrm{~g})$ was calculated from the experimental pole figures where the orientation $\mathrm{g}$ is given in terms of the Euler angles (Fig. 6). The calculated ODF figures show that the texture of the investigated material exhibits a well developed y-fibre [uvw] $\{111\}$ with a spread towards the $\alpha$-fibre [110] $\{h k l\}$ as indicated in the $\varphi_{2}=45^{\circ}$ section of the Euler space. As previously proposed by Bacroix et al. (1994), the orthotropic symmetry is not assumed in the ODF calculation in order to facilitate the comparison between initial and induced textures.

\section{THE CONSTITUTIVE LAWS AND THEIR IDENTIFICATION}

An accurate description of the material's behaviour is required to obtain valuable FE predictions in forming processes. From a numerical point of view, focus should be dedicated to the yield locus defining the plastic behaviour of the material. The initial yield locus as well as its evolution during plastic deformation must be accurately captured by the constitutive law. It is indeed well-known that the plastic deformation of polycrystalline materials induces reorientation of individual grains into preferred orientations. This phenomenon, i.e. texture evolution, is responsible for induced mechanical anisotropy of material, which plays an important role in forming processes. The implementation of the texture evolution into FE codes is therefore of great importance. Unfortunately, micro-macro models generally require very long computation time and large memory storage. These considerations led ArGEnCo team to the development of a local yield locus approach able to predict texture evolution during FE modelling of industrial forming processes. With this model, only a small zone of the yield locus is computed. This zone is updated when its position is no longer located in the part of interest in the yield locus or when the yield locus changes due to texture evolution.

This paper mainly focuses on three different description of the yield surface: the quadratic Hill-1948 yield surface, the so-called MINTY and EVOL yield surfaces (see Sections 3.2 and 3.3). The EVOL and MINTY local yield locus approaches are based on crystal plasticity computation respectively with and without taking into account the crystallo-graphic texture evolution. 
Fig. 6: Initial texture of the If mild steel. Sections of the Euler space.

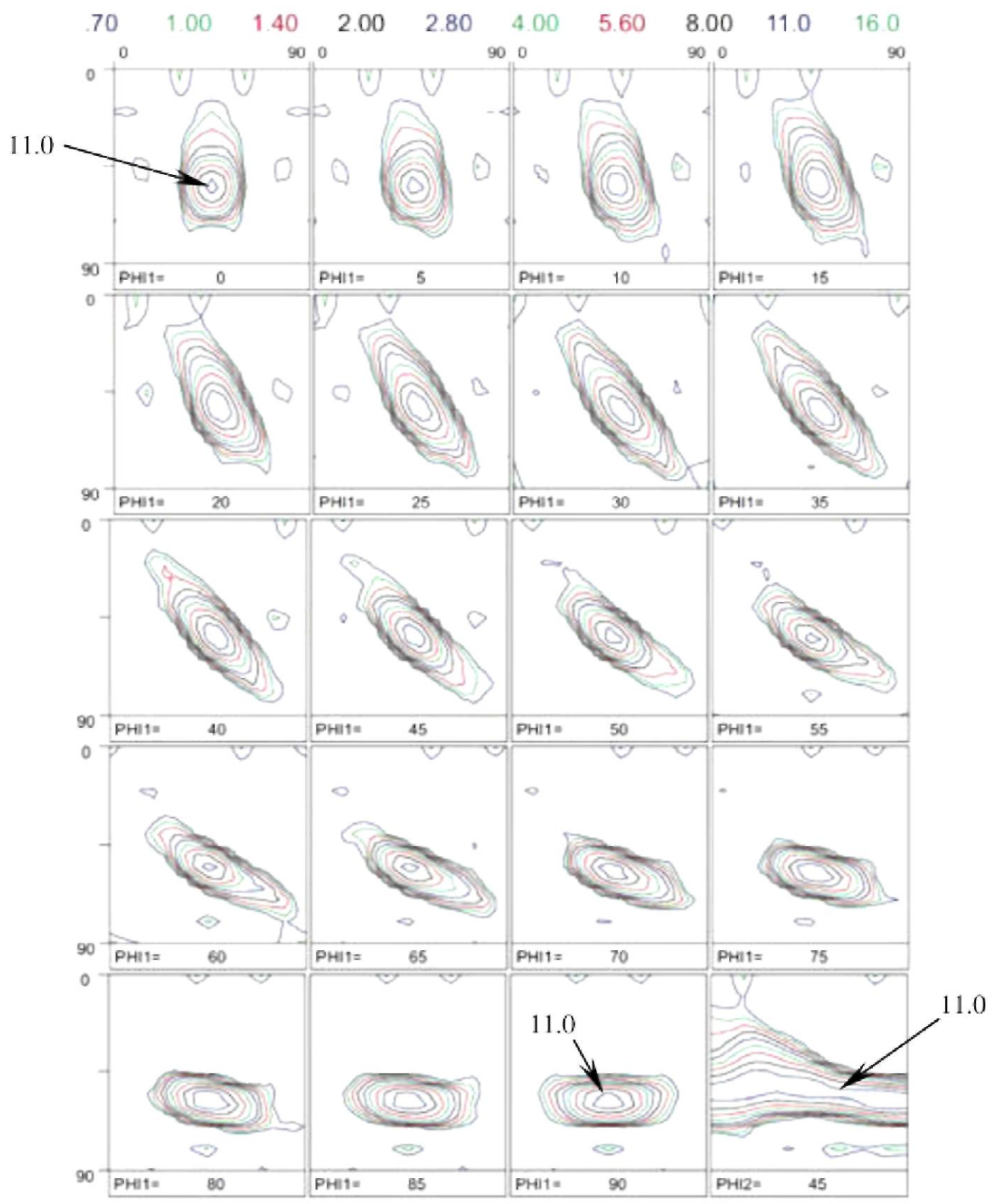

\subsection{Hill-1948}

The classical quadratic (Hill, 1948) criterion was used. Hill-1948's yield locus is largely implemented in several finite element codes and then allows the comparison of different FE codes eliminating the influence of the yield locus formulation. Hill-1948's yield locus is defined by

$$
f=\sqrt{\boldsymbol{\sigma}^{\mathrm{T}}: \mathbf{H}: \boldsymbol{\sigma}}-\sigma_{\mathrm{F}}=0
$$

where $\sigma$ is the stress state expressed as a six-component vector; $\sigma_{\mathrm{F}}$ is the flow stress whose evolution describes the isotropic work hardening. In the present work, the following classical law is adopted

$$
\sigma_{\mathrm{F}}=C\left(\varepsilon_{0}+\bar{\varepsilon}^{p}\right)^{n}
$$


This formulation expresses the flow stress as a function of the equivalent plastic strain $\bar{\varepsilon}^{p}$. C, $\varepsilon_{0}$ and $n$ are material parameters. $\mathrm{H}$ is the matrix containing Hill parameters describing the material anisotropy. It is written in the form

$$
\mathbf{H}=\frac{1}{2}\left(\begin{array}{cccccc}
G+H & -H & -G & 0 & 0 & 0 \\
-H & H+F & -F & 0 & 0 & 0 \\
-G & -F & F+G & 0 & 0 & 0 \\
0 & 0 & 0 & 2 N & 0 & 0 \\
0 & 0 & 0 & 0 & 2 L & 0 \\
0 & 0 & 0 & 0 & 0 & 2 M
\end{array}\right) .
$$

The six parameters $F, G, H, N, L$ and $M$ of this matrix must be adjusted to correctly fit material's behaviour. Different possibilities can be considered. The fitting can be achieved thanks to experimental yield stress measurements. It can also be based on strain measurements. In the current case the Hill's parameters are computed from the Hill's coefficients of anisotropy $r_{\alpha}$, i.e. ratio of width plastic strain rate to thickness plastic strain rate during uniaxial tensile tests; $\alpha$ is the angle between the initial rolling direction and the loading direction.

\subsection{MINTY}

This local yield locus approach has already been validated and extensively used to model deep drawing processes (see Duchêne, 2003; Habraken and Duchêne, 2004 for details). This model is specific in the sense that it does not use an analytical yield locus formulation either for plastic criterion or in the stress integration scheme. A linear stress-strain interpolation in the five-dimensional (5D) stress space described by Eq. (8) is used at the macroscopic scale:

$$
\boldsymbol{\sigma}_{v}=\tau \mathbf{C}: \mathbf{u}
$$

In this equation, $\sigma_{v}$ is a $5 \mathrm{D}$ vector containing the deviatoric part of the stress tensor; the hydrostatic part being elastically computed according to Hooke's law. The $5 \mathrm{D}$ vector $\mathrm{u}$ is the deviatoric plastic strain rate direction (it is a unit vector), $\tau$ is a scalar describing the work hardening according to Swift's type exponential relationship of Eq. (9), where the strength coefficient $K$, the offset $\Gamma^{0}$ and the hardening exponent $n$ are material parameters fitted on experimental data and $\Gamma$ is the polycrystal induced slip.

$$
\tau=K \cdot\left(\Gamma^{0}+\Gamma\right)^{n}
$$

This formulation is equivalent to Eq. (6) but expressed at the microscopic scale. The parameters $K, \Gamma^{0}$ and $n$ can easily be obtained from the macroscopic parameters $\mathrm{C}, \varepsilon_{0}$ and $n$ of Eq. (6) thanks to the so-called Taylor's factor.

The macroscopic anisotropic interpolation is included in matrix $\mathrm{C}$ of Eq. (8). Its identification relies on five directions: $u_{i}(i=1 \ldots 5)$ advisedly chosen in the deviatoric strain rate space and their associated deviatoric stresses: $\sigma_{i}(i=1 \ldots 5)$ computed by the polycrystal plasticity model. These deviatoric stress vectors $\sigma_{i}$ lie on the yield locus. The micro-macro model uses in fact Taylor's assumption of equal macroscopic strain rate and microscopic crystal strain rate tensors. It computes the average of the response of a set of representative crystals evaluated with a microscopic model taking into account the plasticity at the level of the slip systems. In this paper, the rate insensitive Full Constraints (FC) Taylor's model is investigated. Details about microscopic Taylor type models as well as about micro-macro transition assumptions can be found in Habraken and Duchêne (2004).

The deviatoric stress vectors $\sigma_{i}$ define the vertices of the interpolation domain and are called 'stress nodes'. The C matrix is built on the basis of these five stress nodes. With this method, only a small part of the yield locus is known. As long as the interpolation is achieved in the domain delimited by these five stress nodes, the interpolation matrix $\mathrm{C}$ is valid. When the stress direction explored during FE computation falls out of this domain, updating of the stress nodes must take place; a new interpolation matrix is then computed. The above considerations are sufficient to understand the basic concepts of the local yield locus implemented in Lagamine FE code (see Section 5.1). Further details and properties of such parameterisation of an $\mathrm{N}$-dimensional space have been investigated in Duchêne (2003) and Habraken and Duchêne (2004). 


\subsection{EVOL}

While 'MINTY' refers to the local yield locus approach without computation of texture evolution (the initial texture is then used throughout the FE simulation), 'EVOL' refers to the same approach but with activation of the texture evolution. Material's texture is represented at each integration point of the FE mesh by a set of crystallographic orientations. Texture evolution is computed by Taylor's model on the basis of the strain history for each integration point. The set of crystallographic orientations of each integration point is subsequently updated. As it induces an evolution of the material's behaviour, when texture evolution takes place, the $\mathrm{C}$ matrix describing the current local yield locus must be updated. The authors refer to Habraken and Duchêne (2004) for further details about the strategy used for texture evolution.

\subsection{Identification of material parameters}

During this study, a low carbon IF mild steel FeP06t of $0.7 \mathrm{~mm}$ thickness, was analysed. This steel sheet is produced by cold rolling and annealed. In order to achieve numerical simulations with this material, its mechanical properties were determined. The classical isotropic elastic properties of steel were used, as presented in Table 1 .

Hill's parameters describing the anisotropy of the yield locus were determined on the basis of the Hill's coefficients of anisotropy according to Eq. (10)

$$
r_{0}=\frac{H}{G} ; \quad r_{45}=\frac{2 N-(F+G)}{2(F+G)} ; \quad r_{90}=\frac{H}{F},
$$

with the additional assumptions:

$N=L=M$.

Table 1: Elastic properties of FeP06t

\begin{tabular}{ccc}
\hline & Young's modulus (MPa) & Poisson's ratio \\
\hline FeP06t & 210,000 & 0.3 \\
\hline
\end{tabular}

Table 2: Hill's coefficients of anisotropy for FeP06t

\begin{tabular}{cccc}
\hline & $\boldsymbol{r}_{\mathbf{0}}$ & $\boldsymbol{r}_{\mathbf{4 5}}$ & $\boldsymbol{r}_{\mathbf{9 0}}$ \\
\hline FeP06t & 2.53 & 1.84 & 2.72 \\
\hline
\end{tabular}

Table 3: Hill's parameters of FeP06t steel sheet

\begin{tabular}{ccccccc}
\hline & $\boldsymbol{F}$ & $\boldsymbol{G}$ & $\boldsymbol{H}$ & $\boldsymbol{N}$ & $\boldsymbol{L}$ & $\boldsymbol{M}$ \\
\hline FeP06t & 0.53 & 0.57 & 1.43 & 2.56 & 2.56 & 2.56 \\
\hline
\end{tabular}

Furthermore, as $\sigma_{\mathrm{F}}$ in Eq. (5) is supposed to represent the work hardening observed during a uniaxial tensile test along the rolling direction, the additional condition $H+G=2$ must be fulfilled.

The Hill's coefficients of anisotropy along the rolling direction $r_{0}$, at $45^{\circ}$ from the rolling direction $r_{45}$ and along the transverse direction $r_{90}$ are presented in Table 2. The corresponding Hill's parameters are in Table 3.

For the crystal plasticity based constitutive laws, the texture of the FeP06t steel sheet was measured by X-ray diffraction. The resulting pole figures are plotted in Fig. 5. A set of 2000 representative crystallographic orientations was computed from the texture measurements thanks to the software ODFLAM (Van Houtte, 1994). 
The agreement between the phenomenological Hill's yield locus and the one computed by the polycrystal plasticity FC Taylor's model is assessed thanks to comparison of sections of the yield locus for the studied steel. The sections in the plane $\sigma_{1}-\sigma_{2}$ are shown in Fig. 7.

The bi-axial and plane strain fields show quite large discrepancy. The latter is explained by the limitation of the quadratic criterion in describing the anisotropy of the mild steel. However, in the stress area involved in the present work (i.e. pure shear zone) the yield loci are quite similar for both models.

Finally, Table 4 presents the hardening parameters for both formulations of Swift's hardening model: microscopic formulation with Eq. (9) and macroscopic formulation with Eq. (6). These parameters were fixed from shear test experiments.

Fig. 7: Section of the initial yield locus of FeP06t material with Taylor-Bishop-Hill (TBH) model and with Hill criterion, both coupled to an isotropic hardening.

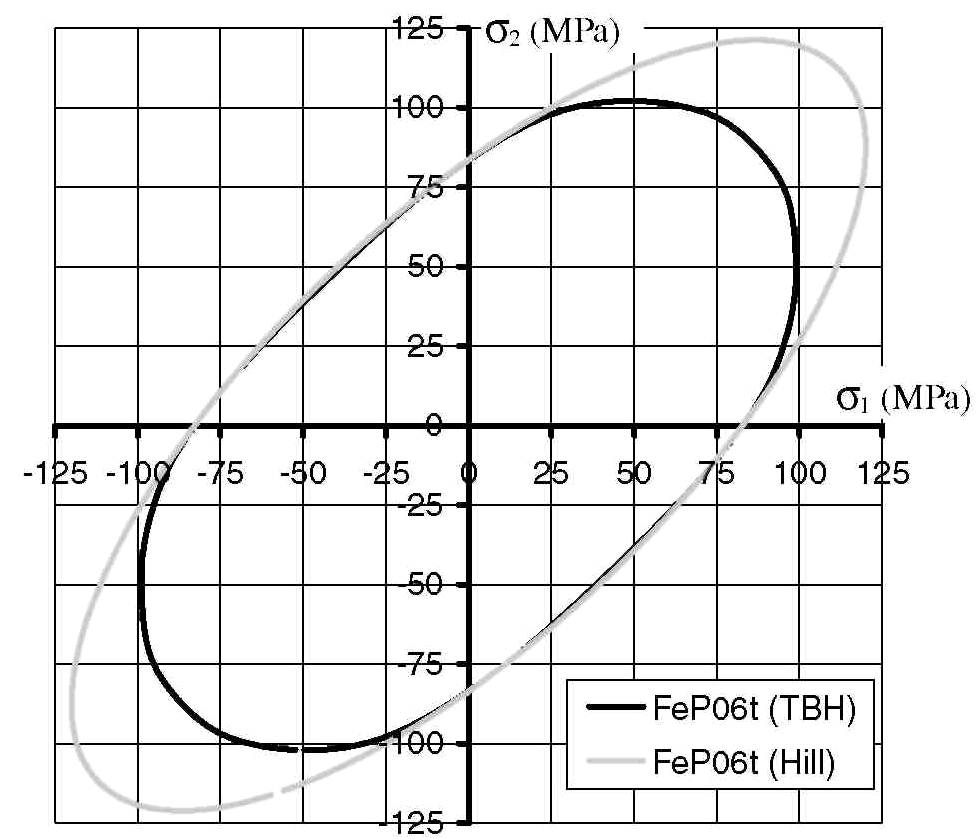

Table 4: Hardening parameters of FeP06t for the formulations of Eqs. (9) and (6)

\begin{tabular}{ccccccc}
\hline & \multicolumn{3}{c}{$\sigma_{\mathrm{F}}=C\left(\varepsilon_{0}+\bar{\varepsilon}^{p}\right)^{n}$} & \multicolumn{3}{c}{$\boldsymbol{\tau}=\boldsymbol{K}\left(\boldsymbol{\Gamma}^{\mathbf{0}}+\boldsymbol{\Gamma}\right)^{\boldsymbol{n}}$} \\
\cline { 2 - 7 } & $\boldsymbol{C}(\mathbf{M P a})$ & $\boldsymbol{\varepsilon}_{\mathbf{0}}$ & $\boldsymbol{n}$ & $\boldsymbol{K}(\mathbf{M P a})$ & $\boldsymbol{\Gamma}^{\mathbf{0}}$ & $\boldsymbol{n}$ \\
\hline FeP06t & 550.3 & 0.0011 & 0.278 & 143.67 & 0.00315 & 0.278 \\
\hline
\end{tabular}

\section{The three investigated rotation methods}

Beside material's behaviour description, it appeared that the finite element formulation has also a large influence on the accuracy of the simulation results. Two finite element types were examined during this study: the BWD3D and the JET3D elements, described below. These elements belong to the same family of 8-node 3D brick elements with a mixed formulation and one integration point. The main differences between the BWD3D and the JET3D elements are the method used to avoid shear locking and the definition of the local reference axes (see Fig. 1) used to integrate the constitutive law. A particular attention is paid to the description of the three methods used in these elements for the computation of the local reference axes rotation during FE simulations. These two 3D elements have already been compared during deep drawing simulations in Duchêne and Habraken (2005). 
The rotation methods proposed hereafter are implemented in an updated Lagrangian FE framework. Small time steps are therefore employed so that particular assumptions can be done in order to simplify the integrations of rotation rates (spins) over each time step.

\subsection{The Jaumann type approach and the BWD3D finite element}

The first method used to compute the rotation of the local frame is a Jaumann type approach. This method is called "Constant Symmetric Local Velocity Gradient" (CSLVG) and is coupled with the BWD3D finite element. This element is based on the nonlinear three-field (stress, strain and displacement) Hu-Washizu variational principle (Belytschko and Bindeman, 1991).

A first feature of the BWD3D element is a new shear locking treatment based on the Wang-Wagoner method (Wang and Wagoner, 2004). This method identifies the hourglass modes responsible of the shear locking and removes them. The two bending hourglass modes and the warp (non-physical) hourglass mode are eliminated. The volumetric locking treatment is also based on the elimination of inconvenient hourglass modes. The WangWagoner method, contrarily to some other shear locking methods (see e.g. Li and Cescotto, 1997), has deep physical roots which makes it very efficient for various FE analyses.

A second feature of this new element is the use of a co-rotational reference system. In order to be able to identify the hourglass modes (which is a crucial point of the method), the formulation of the element kinematics must be expressed in a co-rotational reference system (Belytschko and Bindeman, 1991), closely linked to the element coordinates. This reference system must have its origin at the center of the element and its reference axes are aligned (as much as possible, depending on the element shape) with element edges. A grateful consequence of this co-rotational reference system is a simple and accurate treatment of the hourglass stress objectivity, by using initial and final time step rotation matrices. Further details about the hourglass and the locking treatments in the BWD3D element can be found in Duchêne et al. (2005).

In the element name, 'B' stands for Belytschko (Belytschko and Bindeman, 1991); 'W' stands for Wang and Wagoner (Wang and Wagoner, 2004); 'D' is for Duchêne and de Montleau (Duchêne et al., 2005) and, finally, '3D' is for three dimensions. Up to now, the BWD3D element has proved its superiority in deep drawing simulations, incremental forming and large strain torsion (see Duchêne et al., 2007). This new finite element was successfully compared to its former version: the BLZ3D (Zhu and Cescotto, 1994) where another shear locking treatment was used. This proves the large influence of the accuracy of the element formulation and in particular its shear locking treatment on FE results.

An important aspect of the BWD3D element is the CSLVG method used to determine the local reference axes. This method is largely described here because it is one of the main points of this paper. Note that this local reference system is necessary to ensure the objectivity during the integration of the constitutive law and it is absolutely independent from the co-rotational reference system used to identify the hourglass modes. At a first stage, all vectors and tensors are computed in the global reference system, which remains fixed during the deformation of the solid. The kinematics in continuum mechanics involves the computation of the deformation gradient tensor:

$$
\mathbf{F}=\frac{\partial \mathbf{x}}{\partial \mathbf{X}}
$$

where $\mathrm{x}=\mathrm{x}(\mathrm{X}, t)$ is the mapping of the initial configuration of the solid, having coordinates $\mathrm{X}$, to the current configuration at time $t$. The velocity gradient in the current configuration is computed by Eq. (13).

$$
\mathbf{L}=\frac{\partial \dot{\mathbf{x}}}{\partial \mathbf{x}}=\dot{\mathbf{F}} \mathbf{F}^{-1}
$$

The symmetric part and the skew-symmetric part of $\mathrm{L}$ are respectively the well-known strain rate tensor and the spin tensor, related to rigid body rotation.

The implementation of a nonlinear constitutive law in a large strain FE code implies a step by step procedure. The integration of the kinematics equations must be achieved carefully due to the incremental procedure.

The configuration at the beginning of one step is called $A$ at time $t_{\mathrm{A}}$ and the configuration at the end of the step is $B$ at time $t_{B}$. During the computation of one FE time step, the velocity field at the beginning of the step is known 
and an estimation of the velocity field at the end of the step is assumed. The deformation gradient tensors at the beginning and the end of the step are computed:

$$
\mathbf{F}_{A}=\frac{\partial \mathbf{x}_{A}}{\partial \mathbf{X}} \quad \text { and } \quad \mathbf{F}_{B}=\frac{\partial \mathbf{x}_{B}}{\partial \mathbf{X}}
$$

The incremental deformation gradient tensor for the considered step is

$$
\mathbf{F}_{A B}=\mathbf{F}_{B} \mathbf{F}_{A}^{-1}=\frac{\partial \mathbf{x}_{B}}{\partial \mathbf{x}_{A}}
$$

According to this incremental process, the configurations at the beginning and the end of the step are known but a strain path must be chosen between these two configurations. Different assumptions have been examined by several authors (see e.g. Hughes, 1983; Ponthot, 2002). The method used to determine the strain path in the BWD3D element was developed by Cescotto (1984) and Munhoven and Habraken (1995). It is called Constant symmetric local velocity gradient. The constancy assumption of the velocity gradient imposes the strain path. According to Eq. (13), the deformation gradient tensor must satisfy the following differential equation

$$
\mathbf{L}=\dot{\mathbf{F}}(t)[\mathbf{F}(t)]^{-1}=\text { constant }, \quad t \in\left[t_{A}, t_{B}\right]
$$

with the initial condition

$$
\mathbf{F}\left(t_{A}\right)=\mathbf{F}_{A}
$$

The following solution fulfils Eqs. (16) and (17)

$$
\mathbf{F}(t)=\exp \left(\mathbf{L}\left(t-t_{A}\right)\right) \mathbf{F}_{A}
$$

The additional condition $\mathrm{F}\left(t_{\mathrm{B}}\right)=\mathbf{F}_{B}$ allows finding the constant value of the velocity gradient

$$
\mathbf{L}=\frac{1}{\Delta t} \ln \left(\mathbf{F}_{A B}\right)
$$

where $\Delta t=t_{B}-t_{A}$ is the size of the time step.

The velocity gradient computed with Eq. (19) is constant during the time step but it is generally non-symmetric as $F_{A B}$ is not necessary symmetric. The symmetry of the velocity gradient can however be obtained by expressing it in another reference system. In other words, the symmetry condition in this method fixes the choice of the local reference system.

Let us now present how to determine the local reference system and the velocity gradient according to the symmetry condition. All vectors and tensors expressed in the local reference system are noted with a'. First, the deformation gradient tensor expressed in the local reference system is obtained thanks to Eq. (20).

$$
\mathbf{F}^{\prime}=\frac{\partial \mathbf{x}^{\prime}}{\partial \mathbf{X}^{\prime}}=\frac{\partial \mathbf{x}^{\prime}}{\partial \mathbf{x}} \frac{\partial \mathbf{x}}{\partial \mathbf{X}} \frac{\partial \mathbf{X}}{\partial \mathbf{X}^{\prime}}=\mathbf{R}^{\mathrm{T}} \mathbf{F} \mathbf{R}_{0}
$$

where $\mathrm{R}$ is the current rotation matrix between the local and the global reference systems (the orthogonal condition of rotation matrices implies $\mathrm{R}^{-1}=\mathrm{R}^{\mathrm{T}}$ ). $\mathrm{R}_{0}$ is the corresponding rotation matrix at the beginning of the process. Due to the step by step procedure of the FE code, the rotation matrix $\mathrm{R}$ has the value $R_{A}$ at the beginning of the step and $R_{B}$ at the end of the step. Indeed, while the global reference system remains always fixed, the local one evolves during the time step. The incremental rotation of the local reference system during the time step is simply $R_{B} R_{A}{ }^{T}$. 
Similarly to Eq. (15), the incremental deformation gradient expressed in the local reference system is

$$
\mathbf{F}_{A B}^{\prime}=\mathbf{F}_{B}^{\prime} \mathbf{F}_{A}^{\prime-1}=\frac{\partial \mathbf{x}_{B}^{\prime}}{\partial \mathbf{x}_{A}^{\prime}}
$$

The local velocity gradient can then be obtained

$$
\mathbf{L}^{\prime}=\frac{1}{\Delta t} \ln \left(\mathbf{F}_{A B}^{\prime}\right)
$$

This velocity gradient is constant during the time step and it is furthermore assumed to be symmetric. Therefore, Eq. (22) implies that $\mathrm{F}_{A B}^{\prime}$ is also symmetric. Making use of Eq. (20), allows developing the formulation of the local incremental deformation gradient

$$
\mathbf{F}_{A B}^{\prime}=\mathbf{F}_{B}^{\prime} \mathbf{F}_{A}^{\prime-1}=\left(\mathbf{R}_{B}^{\mathrm{T}} \mathbf{F}_{B} \mathbf{R}_{0}\right)\left(\mathbf{R}_{0}^{\mathrm{T}} \mathbf{F}_{A}^{-1} \mathbf{R}_{A}\right)=\mathbf{R}_{B}^{\mathrm{T}} \mathbf{F}_{B} \mathbf{F}_{A}^{-1} \mathbf{R}_{A}=\mathbf{R}_{B}^{\mathrm{T}} \mathbf{F}_{A B} \mathbf{R}_{A} .
$$

At this stage, $\mathrm{F}^{*}{ }_{A B}$ is defined by

$$
\mathbf{F}_{A B}^{*} \triangleq \mathbf{F}_{A B} \mathbf{R}_{A}=\mathbf{R}_{B} \mathbf{F}_{A B}^{\prime}
$$

where the second equality derives from Eq. (23). Finally, Eq. (22) yields to

$$
\begin{aligned}
\mathbf{L}^{\prime} & =\frac{1}{\Delta t} \ln \left(\mathbf{F}_{A B}^{\prime}\right)=\frac{1}{2 \Delta t} \ln \left(\mathbf{F}_{A B}^{\prime} \mathbf{F}_{A B}^{\prime}\right)=\frac{1}{2 \Delta t} \ln \left(\left(\mathbf{F}_{A B}^{\prime}\right)^{\mathrm{T}} \mathbf{F}_{A B}^{\prime}\right) \\
& =\frac{1}{2 \Delta t} \ln \left(\left(\mathbf{R}_{B}^{\mathrm{T}} \mathbf{F}_{A B}^{*}\right)^{\mathrm{T}}\left(\mathbf{R}_{B}^{\mathrm{T}} \mathbf{F}_{A B}^{*}\right)\right)=\frac{1}{2 \Delta t} \ln \left(\left(\mathbf{F}_{A B}^{*}\right)^{\mathrm{T}} \mathbf{R}_{B} \mathbf{R}_{B}^{\mathrm{T}} \mathbf{F}_{A B}^{*}\right) \\
& =\frac{1}{2 \Delta t} \ln \left(\left(\mathbf{F}_{A B}^{*}\right)^{\mathrm{T}} \mathbf{F}_{A B}^{*}\right) .
\end{aligned}
$$

The symmetry condition of $\mathrm{F}_{A B}^{\prime}$ has been used in the above developments, while this last formulation of the local velocity gradient is checked to be effectively symmetric. The implementation of this method in a FE code is achieved according to the following algorithm:

- All variables at the beginning of the step (state $A$ ) are known (expressed in the global reference system) according to previous step computations.

- With the estimation of the velocity field at the end of the step, $\mathrm{F}_{A B}$ is computed according to Eqs. (14) and (15).

- The definition of $\mathrm{F}^{*}{ }_{A B}$ is exploited using the first equality of Eq. (24).

- The constant symmetric local velocity gradient L' is computed according to the last equality of Eq. (25).

- The stress tensor at the beginning of the step is expressed in the local reference system according to

$\boldsymbol{\sigma}_{A}^{\prime}=\mathbf{R}_{A}^{\mathrm{T}} \boldsymbol{\sigma}_{A} \mathbf{R}_{A}$

- The constitutive law is called to achieve the stress integration on the current time step

$$
\boldsymbol{\sigma}_{B}^{\prime}=\boldsymbol{\sigma}_{A}^{\prime}+f\left(\boldsymbol{\sigma}_{A}^{\prime}, \mathbf{L}^{\prime}, \Delta t, \text { internal variables }\right) .
$$

Conveniently, the incremental objectivity is automatically satisfied thanks to the computation in the local reference system. As a consequence, Jaumann type corrections are intrinsically included in the formulation and need not to be added to the natural derivatives during the stress integration by the constitutive law. 
- The right decomposition of $\mathrm{F}^{*}{ }_{A B}$ yields to

$$
\mathbf{F}_{A B}^{*}=\mathbf{R}^{*} \mathbf{U}^{*}
$$

Identification of Eq. (28) and the second equality of Eq. (24) allows determining $R_{B}$. Indeed, bearing in mind that $\mathrm{F}_{A B}^{\prime}$ is symmetric, we have $\mathrm{R}_{B}=\mathrm{R}^{*}$ and incidentally $\mathrm{F}_{A B}^{\prime}=\mathrm{U}^{*}$.

- Finally, the stress tensor at the end of the step can be computed by

$$
\boldsymbol{\sigma}_{B}=\mathbf{R}_{B} \boldsymbol{\sigma}_{B}^{\prime} \mathbf{R}_{B}^{\mathrm{T}}
$$

\subsection{The co-rotational approach and the JET3D finite element}

The second method used to determine the local frame during FE simulations in based on a co-rotational approach and is coupled with the JET3D finite element. In the manner of the BWD3D, the JET3D element is an 8-node 3D brick element with mixed formulation adapted to large strains and large displacements. It uses a reduced integration scheme (i.e. with only one integration point) and an hourglass control technique. It is based on the $\mathrm{Hu}-$ Washizu principle with the "assumed strain method". Further details can be found in Li et al. (1995) and Li and Cescotto (1997). The main differences between the BWD3D and the JET3D elements are:

- The shear locking and the hourglass modes treatment: instead of removing totally undesirable hourglass modes (the Wang-Wagoner method used in the BWD3D), three distinct $\beta$ parameters $(\in[0,1])$ are introduced to reduce the deformation energy associated to the whole set of hourglass modes. Two kinds of contradictory problems can occur with hourglass and locking treatment: (1) Bending dominated problems where the excessive shear energy associated to hourglass modes should be removed by setting a value near zero for $\beta$; (2) Torsion dominated problems where only shear energy exists. In this case, a $\beta$ value around one should be used to avoid unwanted torsion type hourglass modes. Three distinct $\beta$ values are introduced in the formulation to allow a different behaviour of the element along the three reference directions ( $X, Y$ and $\mathrm{Z}$ ). The $\beta$ values can either be imposed by the FE code user, or be determined automatically by the code according to the aspect ratio of the considered element along the three reference axes.

- The choice of the local reference system for the objective integration of the constitutive law. A co-rotational reference system is defined in a similar way than in the BWD3D formulation. But, while in the BWD3D element it is just used for the identification of the hourglass modes, the co-rotational reference system of the JET3D element is used as local reference system. As a consequence, the local reference system used to express the constitutive law does not derive from the kinematics of the element formulation but is directly linked to the nodal coordinates. The orientation of the local frame at the end of the FE time step is deduced from the evolution of the nodes positions during the step.

\subsection{The Mandel spin approach}

The co-rotational approach implemented in the JET3D element (Section 4.2) is based on the geometric configuration of the finite element, while the Jaumann type approach of the BWD3D element (Section 4.1) is based on the kinematics of the element. These two methods are independent of the constitutive law used to model the material behaviour. The Mandel spin approach described here is quite different in the sense that it is linked to the material model and especially to the crystals constituting the studied poly-crystal. The macroscopic rotation of the material is computed from the rotation of each crystal. Fig. 8 schematically represents the lattice rotations of three crystals. These rotations correspond to $\omega^{\text {lattice }}$ in Fig. 2.

Due to its cubic symmetry, the crystal lattice must always remain orthogonal (in a relaxed configuration). The crystal rotations during plastic straining are therefore defined unquestionably. The problem of the nonorthogonal axes $x^{\prime}$ and $y^{\prime}$ in Fig. 1 is avoided if crystal rotations are focused on.

In order to have a representation of the crystal rotations, the Mandel spin approach requires the use of a constitutive law based on texture analysis with computation of the texture evolution during plastic deformations. In Lagamine FE code, the Mandel spin approach must be coupled with EVOL constitutive law (Section 3.3). The orientation of each crystal lattice of EVOL's texture representative set is expressed by Euler angles during the whole finite element simulation. The rotation of every crystal during each finite element time step can then be deduced. 
The main difficulty during the computation of the Mandel spin is the computation of the macroscopic rotation of the polycrystal from the rotations of all crystals representative of the volume element. A mean rotation must be computed from crystal rotations. In the particular case where the rotation axis is identical for all the single crystals, the mean rotation can simply be computed as the mean rotation angle around this common rotation axis This particular case corresponds to the representation in Fig. 8.

Unfortunately, in the general three-dimensional case, the rotation axis is different from one crystal to another. Indeed, during numerical simulations of the simple shear test on FeP06t material, it was noticed that only a few crystals rotate around an axis close to the expected macroscopic rotation axis. The rotation angles are also very different from one crystal to another. The rotation axes of the crystals generally have a small deviation from the macroscopic rotation axis. A typical deviation of around $10^{\circ}$ between the macroscopic and the crystal rotation axes was observed. Some crystals even rotate around axes very different from the macroscopic one.

Therefore, four methods were investigated for the computation of the macroscopic mean rotation during one finite element time step (the reader can also refer to other methods proposed in the literature as Van Houtte (2001)). Two methods use quaternions (Dai, 2006) to represent the crystal rotations and two methods use skewsymmetric spin tensors. The detailed computation for each method is presented hereafter. In these descriptions, quaternions are represented by the vector $\mathrm{Q}$, rotation matrices by $\mathrm{R}$ and spin tensors by $\Omega$. As previously, index $A$ corresponds to the beginning of the time step and $B$ to the end of the time step.

Fig. 8: Schematic representation of crystal lattice rotations during plastic straining.

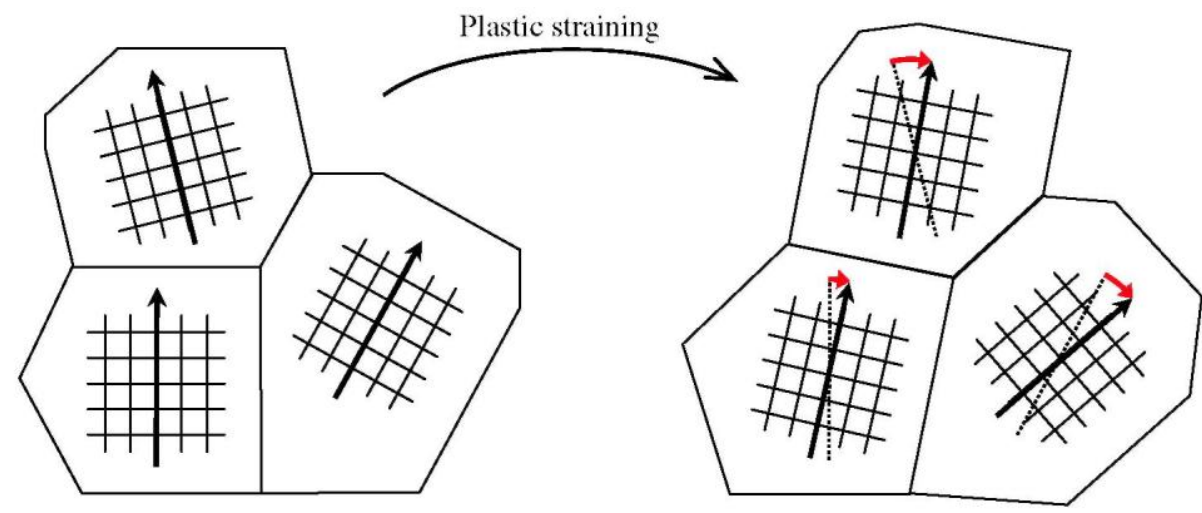

\subsubsection{Method 1}

The mean quaternion for the starting texture (at the beginning of the time step) $\overline{\mathbf{Q}}_{A}$ is computed from the quaternion corresponding to each crystal lattice orientation $\mathbf{Q}_{A}(i)$ :

$$
\overline{\mathbf{Q}}_{A}=\sum_{i=1}^{N_{\text {crys }}} \mathbf{Q}_{A}(i),
$$

where $i$ is the crystal number and $N_{\text {crys }}$ is the total number of crystals included in the texture representative set. Note that $\mathbf{Q}_{A}(\mathrm{i})$ is obtained from the Euler angles of crystal number $i$ at the beginning of the time step: 


$$
\begin{aligned}
\mathbf{Q}_{A}(i)= & {\left[\cos \frac{\varphi_{2}(i)}{2}+\left(\begin{array}{c}
0 \\
0 \\
-\sin \frac{\varphi_{2}(i)}{2}
\end{array}\right)\right]\left[\cos \frac{\phi(i)}{2}+\left(\begin{array}{c}
-\sin \frac{\phi(i)}{2} \\
0 \\
0
\end{array}\right)\right] } \\
& \times\left[\cos \frac{\varphi_{1}(i)}{2}+\left(\begin{array}{c}
0 \\
0 \\
-\sin \frac{\varphi_{1}(i)}{2}
\end{array}\right)\right]
\end{aligned}
$$

The same computation is applied to the texture of the time step end (index $B$ ):

$\overline{\mathbf{Q}}_{B}=\sum_{i=1}^{N_{\text {crys }}} \mathbf{Q}_{B}(i)$.

Then $\overline{\mathbf{Q}}_{A}$ and $\overline{\mathbf{Q}}_{B}$ are normed to represent rotations. Finally the relative quaternion from starting texture to the end texture during the time step is computed by

$$
\overline{\mathbf{Q}}_{A \rightarrow B}=\left(\overline{\mathbf{Q}}_{A}\right)^{-1} \overline{\mathbf{Q}}_{B} .
$$

\subsubsection{Method 2}

The relative rotation during the time step is computed for each crystal in terms of quaternions:

$$
\mathbf{Q}_{A \rightarrow B}(i)=\left(\mathbf{Q}_{A}(i)\right)^{-1} \mathbf{Q}_{B}(i) \text {. }
$$

The mean rotation is computed from the relative rotation of each crystal:

$$
\overline{\mathbf{Q}}_{A \rightarrow B}=\sum_{i=1}^{N_{\text {crys }}} \mathbf{Q}_{A \rightarrow B}(i) .
$$

Finally, $\overline{\mathbf{Q}}_{A \rightarrow B}$ is normed.

\subsubsection{Method 3}

A first step with this method is the computation of the spin tensors corresponding to each crystal $i$. The definition of the spin tensor as a function of one rotation matrix is used:

$$
\mathbf{\Omega}(i)=\dot{\mathbf{R}}(i) \mathbf{R}^{-1}(i) .
$$

Assuming a constant spin tensor during the time increment $\Delta t$, differential Eq. (36) yields:

$$
\mathbf{\Omega}(i)=\frac{1}{\Delta t} \ln [\mathbf{R}(i)]
$$

In this equation, the value of $\Delta t$ must be chosen small enough so that the assumption of a constant spin over the time step is satisfied. The mean spin tensor for the starting texture is computed from the spin tensor of each crystal:

$$
\overline{\mathbf{\Omega}}_{A}=\frac{1}{N_{\text {crys }}} \sum_{i=1}^{N_{\text {crys }}} \boldsymbol{\Omega}_{A}(i) .
$$


The mean rotation matrix of the starting texture is obtained by inverting Eq. (37):

$$
\overline{\mathbf{R}}_{A}=\exp \left(\Delta t \cdot \overline{\mathbf{\Omega}}_{A}\right) .
$$

The same procedure is applied to compute the mean rotation matrix of the texture at the end of the time step: $\overline{\mathbf{R}}_{B}$. And the mean relative rotation of all crystals during the time step is

$$
\overline{\mathbf{R}}_{A \rightarrow B}=\left(\overline{\mathbf{R}}_{A}\right)^{-1} \overline{\mathbf{R}}_{B} .
$$

\subsubsection{Method 4}

The relative rotation of each crystal is computed from its orientation at the beginning and the end of the time step:

$$
\mathbf{R}_{A \rightarrow B}(i)=\left(\mathbf{R}_{A}(i)\right)^{-1} \mathbf{R}_{B}(i)
$$

According to Eq. (37), the relative spin tensor of each crystal is

$$
\mathbf{\Omega}_{A \rightarrow B}(i)=\frac{1}{\Delta t} \ln \left(\mathbf{R}_{A \rightarrow B}(i)\right)
$$

The mean spin tensor rotation is computed from the relative spin tensor of each crystal:

$$
\overline{\mathbf{\Omega}}_{A \rightarrow B}=\frac{1}{N_{\text {crys }}} \sum_{i=1}^{N_{\text {crys }}} \boldsymbol{\Omega}_{A \rightarrow B}(i) .
$$

Finally, the mean relative spin tensor is converted into a rotation matrix:

$$
\overline{\mathbf{R}}_{A \rightarrow B}=\exp \left(\Delta t \cdot \overline{\mathbf{\Omega}}_{A \rightarrow B}\right)
$$

Table 5 summarizes the mains characteristics of the four methods.

These methods provide the rotation of the Mandel frame during one FE time step (integration was achieved when spin tensors were used). During the FE simulations, the mean relative rotation of all crystals computed with these four methods is accumulated during the time steps. The accumulated mean rotation from the initial texture (at the beginning of the FE simulation) to the final texture (at the end of the simulation) yields to the rotation of the local frame corresponding to the Mandel spin approach.

Table 5: Description of the four methods used to compute the Mandel spin

\begin{tabular}{cll}
\hline \multirow{2}{*}{ Rotation represented by } & Mean rotation computation on & \\
\cline { 2 - 3 } & Initial and final textures separately & Crystals relative rotation \\
\hline Quaternions & Method no. 1 & Method no. 2 \\
Spin tensors & Method no. 3 & Method no. 4 \\
\hline
\end{tabular}




\section{FEM SIMULATION RESULTS}

\subsection{Lagamine finite element code}

The numerical simulations described in the present paper were achieved thanks to the home-made FE code LAGAMINE. It is an implicit nonlinear FE code with an updated Lagrangian formulation and is adapted to large strains and large displacements. The kinematics of the code, developed in order to ensure objectivity, is described above. The development of this code by the ArGEnCo department began in 1984 for rolling simulations (Habraken et al., 1998). Since then, it has been applied to numerous other forming processes, such as forging (Habraken and Cescotto, 1990), continuous casting (Castagne et al., 2003, 2004), deep drawing (Duchêne et al., 2002), cooling processes (Habraken and Bourdouxhe, 1992; Casotto et al., 2005) and connection (Drean et al., 2002). LAGAMINE has a large element library (e.g. Cescotto and Charlier, 1993; Zhu and Cescotto, 1994, 1995a, Habraken and Cescotto, 1998), as well as numerous constitutive laws (e.g. Zhu and Cescotto, 1995b; Habraken and Duchêne, 2004).

The BWD3D and the JET3D elements presented in Section 4 were tested for the shearing simulations of FeP06t samples. The Hill, Minty and Evol constitutive laws described in Section 3 were investigated.

\subsection{FEM sensitivity study}

The gauge area of the simple shear sample (30 mm length by $3 \mathrm{~mm}$ width and $0.7 \mathrm{~mm}$ thick) was meshed with 8 node brick elements: BWD3D and JET3D. Two different meshes were investigated in the present study. The first mesh consisted in one layer of 707 elements. It is presented in Fig. 9.

As the strain field was expected to be as uniform as possible, a second mesh with only one element having the dimensions of the sample gauge area was also tested.

The deformed mesh with 707 elements is presented in Fig. 10 for a shear strain $\gamma=0.7$. The corresponding shear strain field is presented in Fig. 11. A uniform shearing was observed in a large part of the sample gauge area, while distortions appeared near the edges due to the boundary conditions (Bouvier et al., 2006).

Fig. 9: Finite element mesh with one layer of 707 elements.

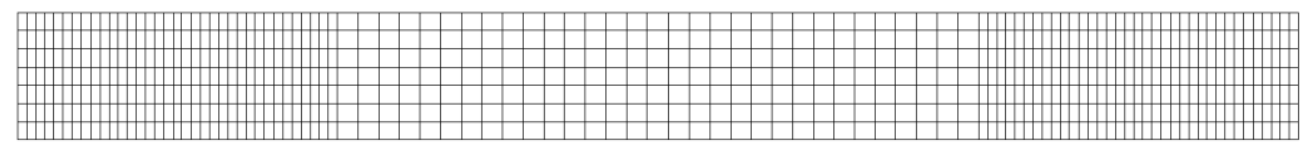

Fig. 10: Deformed mesh for a shear strain $\gamma=0.7$ (JET3D element, Hill law).

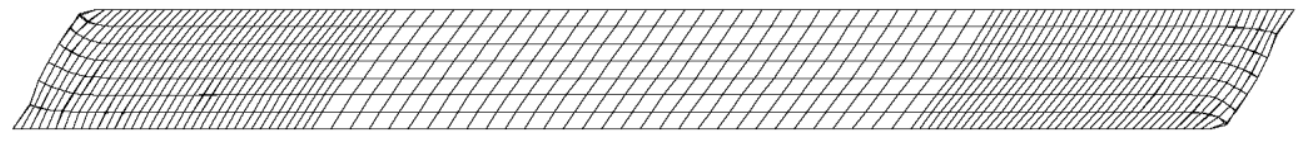

Fig. 11: Shear strain field computed by FEM with 707 elements, JET3D element type and Hill law. The imposed displacement field corresponds to a shear strain $\gamma=0.7$.
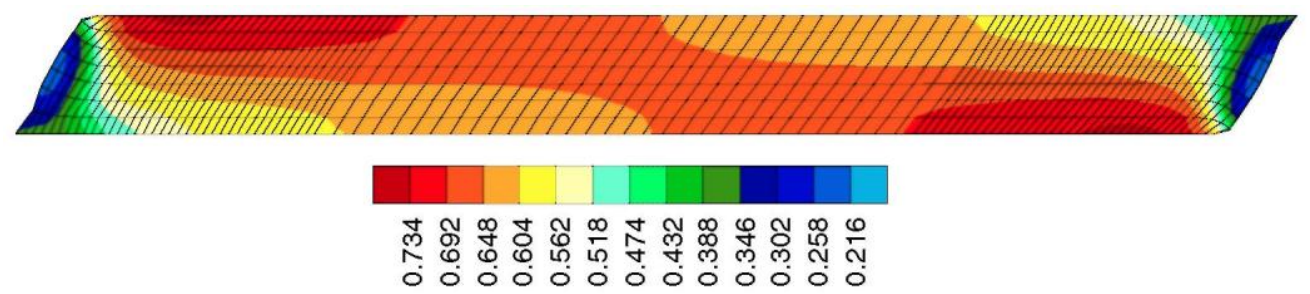

The shear strain distribution computed by FEM (Fig. 11) is in agreement with experimental measurements performed by ARAMIS (Fig. 4). The large uniform shearing zone proves the validity of the computations with only one element. The deformed mesh with one element is completely defined by the boundary conditions and the distribution of the shear strain is imposed to be uniform, without edge effects. 
The evolutions of the shear stress during the deformation with one and 707 elements were compared. As shown in Fig. 12, a good agreement can be noticed. The shear stresses presented in Fig. 12 correspond to the shear stress at the center of the sample, i.e. at the integration point of the unique element for the case with one element and at the integration point of the central element for the case with 707 elements.

\subsection{Experimental and numerical rotation axes}

The main issue of this paper is the analysis of the evolution of the local and material frames (Fig. 1) during a simple shear test using different methods. The experimental texture evolution is also considered as a reference. The crystallographic texture of the deformed samples is measured by X-ray diffraction. (110) and (100) experimental pole figures are presented in Fig. 13. The resulting texture anisotropy is more pronounced with an increase of the intensity of the maxima of the ODFs. A severe deviation from the initial texture is observed consistent with the effect of simple shear test on texture evolution. This mechanical test is known to introduce large rotations of the crystallographic directions of the individual grains towards specific final orientations. Such rotations lead to some loss of the initial orthotropy symmetry of the sheet material (Bacroix et al., 1994; Bacroix and $\mathrm{Hu}, 1995$; Peeters et al., 2001). Based on the experimental deformed texture, among the three symmetry axes of rolled material (i.e. RD, TD and ND), only the ND axis is preserved. Despite this loss of "perfect" orthotropy, near-orthotropy axes are sought as previously proposed by Peeters et al. (2001), by rotating \{RD,TD axes along ND such as the pole figures are as symmetric as possible with respect to these axes. The obtained orthotropy axes on the deformed texture are drawn in Fig. 13. They are computed by a maximization technique based on ODF C-coefficients using MTM-FHM software (Van Houtte, 1994). Their orientation forms an angle of $27^{\circ}$ with respect the orthotropy axes of the as-received material. This observed rotation of the orthotropy axes during simple shear is coaxial with the rotation of the material frame. The common axis is perpendicular to the plane of the steel sheet. It is worth noting that the measured deformed texture is quite far from any rotation of the initial texture (Fig. 5). Indeed, severe texture evolution is observed.

Fig. 12: Effect of the FE meshing on the shear stress-shear strain curve (JET3D element and Hill law).

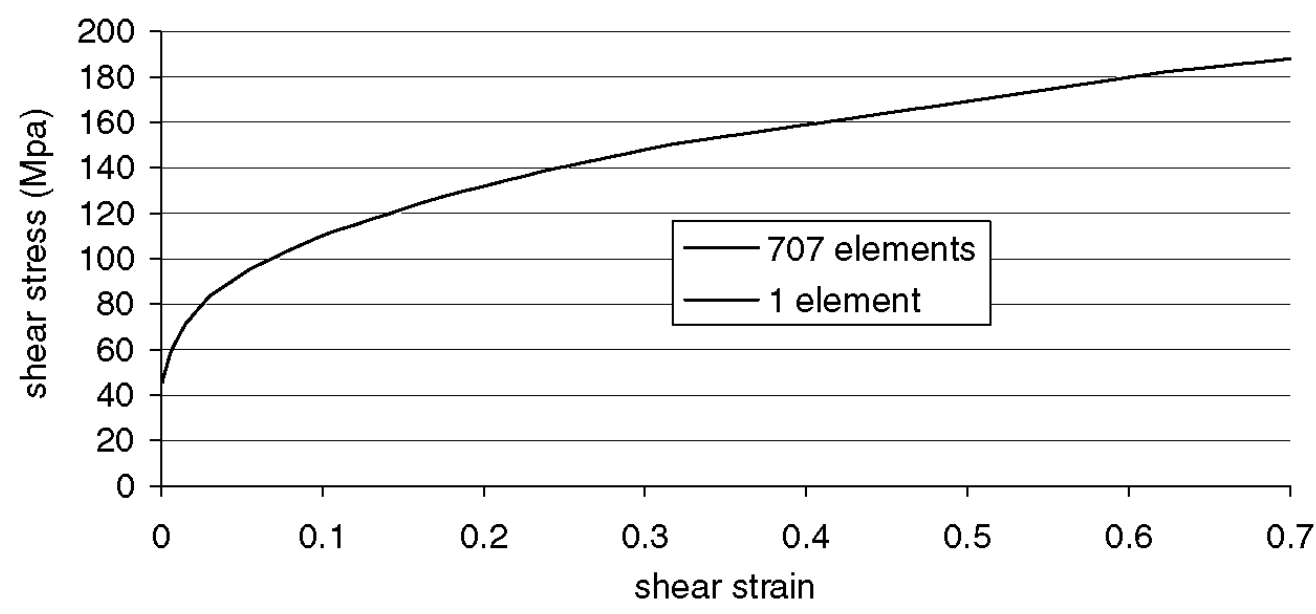

The predicted texture computed with $E v$ ol constitutive law is plotted in Fig. 14. The agreement with experimental measurements is rather good qualitatively (location of the maximum values) and quantitatively (value of the maxima). However, the orientation of the orthotropy axes is slightly different from the experimental one. The rotation angle of the orthotropy axes is about $23^{\circ}$ during the shearing simulation. The discrepancy can partly be explained by the fact that the expected shear strain $\gamma=0.7$, corresponding to $\varphi_{1}=35^{\circ}$ in Fig. 1 , was overreached in the experimental shear test $\left(\varphi_{1}=37.2^{\circ}\right)$.

The three rotation methods presented in Section 4 are applied (i) the "Constant Symmetric Local Velocity Gradient" (CSLVG method) coupled with BWD3D element, (ii) the co-rotational method coupled with JET3D element, (iii) the Mandel spin method. The first method is tested with Hill, Minty and Evol constitutive laws, and with 1 or 707 element(s) for meshing description. The second method is coupled with Hill law and 1 or 707 elements). The Mandel spin is used with only one finite element. 
Fig. 13: The (110) and (100) experimental pole figures resulting from simple shear test along the transverse direction up to 0.7 amount of shear. The near symmetry axes are shown.
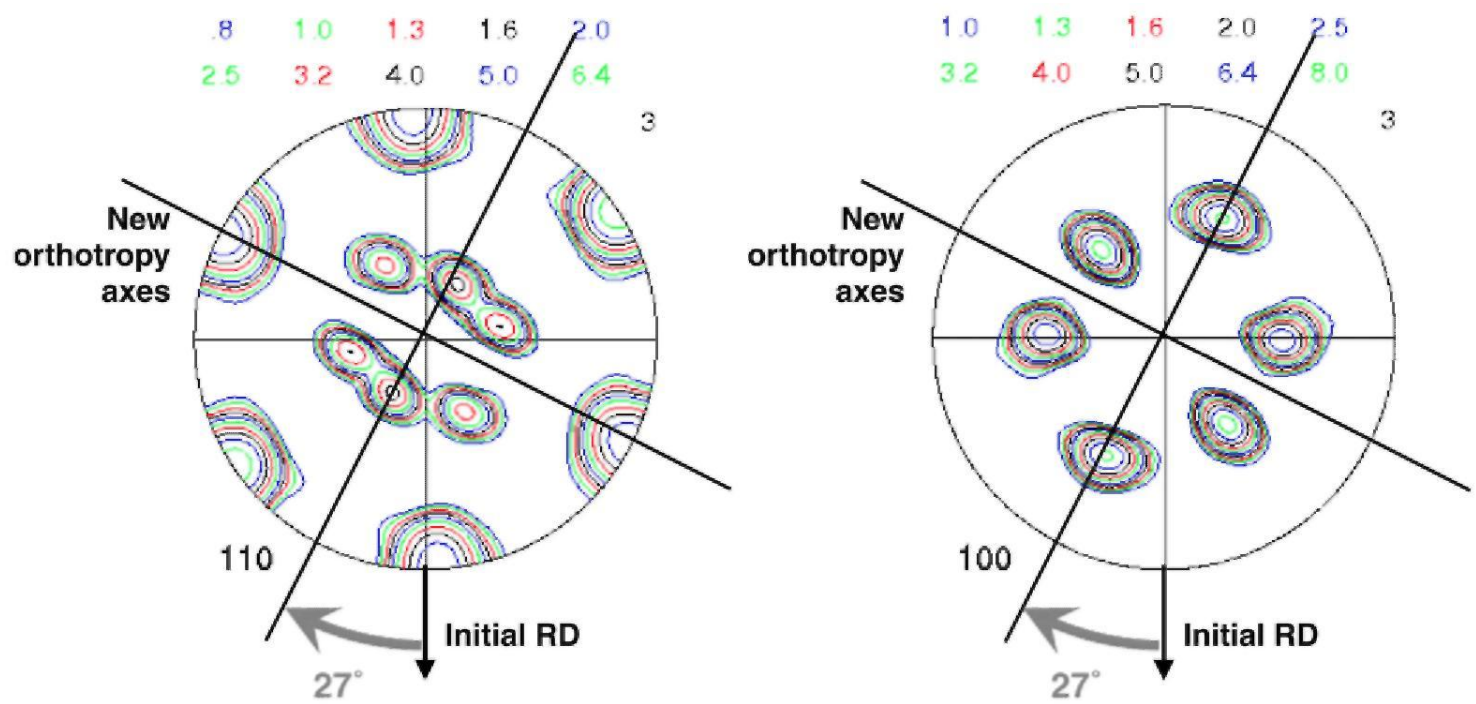

Fig. 14: The (110) and (100) predicted pole figures resulting from simple shear test along the transverse direction up to 0.7 amount of shear. The near symmetry axes are shown.
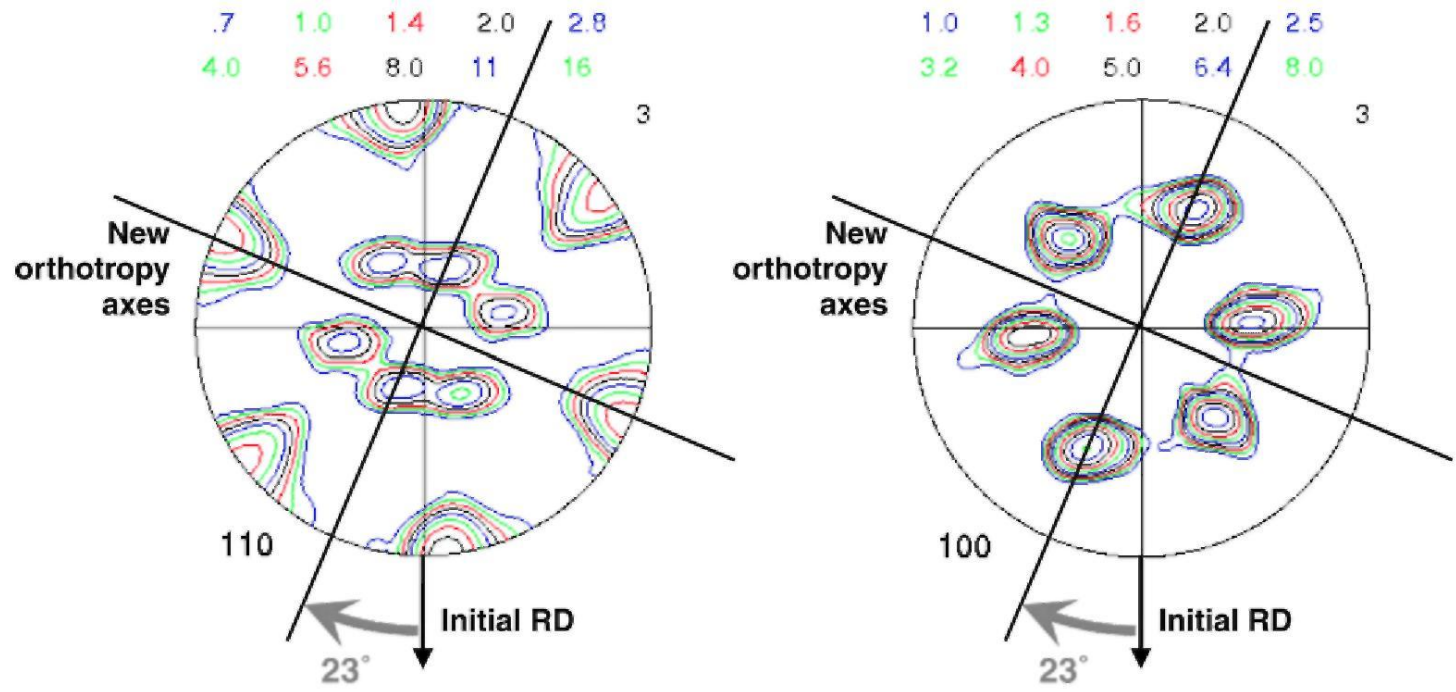

The rotation of the material frame (angle $\varphi_{1}$ in Fig. 1) and the rotation of the local frame (angle $\varphi$ in Fig. 1) computed with the different FE approaches are summarized in Table 6. The experimental and computed rotations of the initial orthotropy axes in the deformed material (Figs. 13 and 14) are also reported in Table 6. Moreover, the rotation of the material frame measured optically by ARAMIS is also provided.

The rotation of the material frame with one BWD3D element was totally imposed by the boundary conditions to a value of $35^{\circ}$, corresponding to a shear strain $\gamma=0.7=\tan \left(35^{\circ}\right)$. As a consequence, the rotation of the local axes was also identical for the three constitutive laws. For the mesh with 707 elements, the rotations were computed for the element at the centre of the sample. Due to the larger freedom of the FE simulations, a slight effect of the constitutive law on the rotation angles can be noticed. For instance, the rotation of $35.2^{\circ}$ of the material frame with Hill law corresponds to a shear strain in the central element of $0.705(0.7 \%$ larger than the imposed global value). This proves again the good homogeneity of the shear strain in the FE simulations. 
The co-rotational approach implemented in the JET3D element was tested with one and 707 elements coupled with Hill constitutive law. As already noticed with the BWD3D element, the rotation of the material frame with one element was imposed by the boundary conditions and a small deviation was observed when a mesh with 707 elements was used ( $1.5 \%$ for $\varphi_{1}$ and $1 \%$ for $\varphi$ ). The rotation of the local frame was computed thanks to geometrical considerations. As shown in Fig. 1, the rotation of $y^{\prime}$-axis was the rotation of the material frame (angle $\varphi_{1}$ ) while $x^{\prime}$ did not rotate. The mean rotation (angle $\varphi_{1} / 2$ ) was used to define the local frame with the corotational approach (see Table 6). The Mandel spin approach was used to compute the mean rotation of the set of crystals representative of the texture with Evol constitutive law. The simulations are achieved with one BWD3D element whose CSLVG method for the computation of the local axes is turned off. The four methods presented in Section 4.3 are tested. Very slight differences are obtained with the different methods adopted in order to compute the macroscopic mean rotation for the Mandel spin. The average values obtained with the different methods are close to the value obtained with the co-rotational approach.

Table 6: Rotation of material and local frames with different approaches

\begin{tabular}{|c|c|c|c|c|c|c|}
\hline & $\begin{array}{l}\text { Rotation analysis } \\
\text { method }\end{array}$ & $\begin{array}{l}\text { Number of } \\
\text { finite elements }\end{array}$ & FE law & $\begin{array}{l}\text { Mean } \\
\text { rotation } \\
\text { computation } \\
\text { method }\end{array}$ & $\begin{array}{l}\text { Rotation of } \\
\text { material frame } \varphi_{1}\end{array}$ & $\begin{array}{l}\text { Rotation of } \\
\text { local frame } \varphi\end{array}$ \\
\hline \multirow{13}{*}{$\begin{array}{l}\text { Numerical } \\
\text { results } \\
\text { imposed shear } \\
\text { strain } \\
\gamma=\tan \left(35^{\circ}\right)\end{array}$} & \multirow{6}{*}{$\begin{array}{l}\text { Jaumann type } \\
\text { approach: CSLVG } \\
\text { with BWD3D } \\
\text { element }\end{array}$} & \multirow[t]{3}{*}{1} & Hill & & $35^{\circ}$ & $20^{\circ}$ \\
\hline & & & Minty & & $35^{\circ}$ & $20^{\circ}$ \\
\hline & & & Evol & & $35^{\circ}$ & $20^{\circ}$ \\
\hline & & \multirow[t]{3}{*}{707} & Hill & & $35.2^{\circ}$ & $20.3^{\circ}$ \\
\hline & & & Minty & & $35.2^{\circ}$ & $20.4^{\circ}$ \\
\hline & & & Evol & & $35^{\circ}$ & $20.2^{\circ}$ \\
\hline & \multirow{6}{*}{$\begin{array}{l}\text { Co-rotational } \\
\text { approach with } \\
\text { JET3D element } \\
\text { Mandel spin } \\
\text { approach }\end{array}$} & 1 & Hill & & $35^{\circ}$ & $17.5^{\circ}$ \\
\hline & & 707 & Hill & & $35.5^{\circ}$ & $17.7^{\circ}$ \\
\hline & & \multirow[t]{4}{*}{1} & \multirow[t]{4}{*}{ Evol } & 1 & & $17.7^{\circ}$ \\
\hline & & & & 2 & & $17.6^{\circ}$ \\
\hline & & & & 3 & & $17.2^{\circ}$ \\
\hline & & & & 4 & & $17.6^{\circ}$ \\
\hline & $\begin{array}{l}\text { Orientation of the } \\
\text { new orthotropy } \\
\text { axes of the } \\
\text { predicted texture }\end{array}$ & 1 & Evol & & & $23^{\circ}$ \\
\hline $\begin{array}{l}\text { Exp. results } \\
\text { actual shear } \\
\text { strain } \\
\gamma=\tan \left(37.2^{\circ}\right)\end{array}$ & $\begin{array}{l}\text { Orientation of the } \\
\text { new orthotropy } \\
\text { axes of the } \\
\text { measured texture } \\
\text { ARAMIS }\end{array}$ & & & & $37.2^{\circ}$ & $27^{\circ}$ \\
\hline
\end{tabular}

\subsection{Discussion}

A first comment about the results presented in Table 6 concerns the discrepancy between the rotation angle $\varphi$ computed by Mandel spin approach (around $17.6^{\circ}$ ) and the rotation of the new orthotropy axes of the predicted texture $\left(23^{\circ}\right)$. A similar observation has been reported in Peeters et al. (2001). It is explained by the fact that the final predicted texture cannot be retrieved by a simple rotation of the initial one. A significant texture evolution can indeed be noticed on the pole figures by comparing Figs. 5 and 14. It was furthermore observed during FE simulations that the rotations at the crystallo-graphic level were very different from one crystal to another, even if the mean rotation axis is the normal direction. As a consequence, the rotation of the orthotropy axes can be significantly different from the mean rotation of the crystals (defining the Mandel spin).

These observations tend to promote the use of a constitutive model able to take into account the texture evolution during the simulations (e.g. Evol law). Such models indeed avoid the representation of the updated texture by just a rotation of the initial one. 
If a constant yield locus shape law is anyway used (Hill or Minty), it is difficult to take into account the evolution of the yield locus just by changing its size (isotropic hardening), its position (kinematic hardening) and its orientation (through the rotation of the local axes). Furthermore, the question about which value of the rotation angle should preferably be used to define the local axes is not obvious. According to Peeters et al (2001), the rotation angle computed by the Mandel spin approach should be regarded as the reference $\left(17.6^{\circ}\right.$ in the present case).

The four methods presented in Section 4.3 to compute the Mandel spin give almost identical results. This statement can be related to the fact that the procedure used to compute the mean rotation of the crystals is applied during each time step on very small rotations, which reduce the influence of the method used. On the other hand, when the Mandel spin approach is applied on large rotations of the crystals, it was found that the four methods provide different results. The mean rotation computed by method 2 remains acceptable while method 3 gives the worst results.

The mean rotation angles of the crystals according to the Mandel spin approach (Table 6) are in agreement with the results of Peeters et al. (2001) obtained during similar numerical simulations of an IF steel sheet submitted to simple shear loading. The rotation axis (normal to the sheet) as well as the rotation angle agree. The Mandel spin approach described in Bacroix et al. (1994) provides larger mean rotation of the crystals $\left(24.5^{\circ}\right.$ for an amount of shear strain $\gamma=\sqrt{3} / 2$ ). However, as reported by Peeters et al. (2001), the rotation of the crystals during shearing is largely dependent to the initial texture of the tested material through the stability of the different texture components with respect to the imposed deformation mode.

Larger amount of shear strains y up to 3.5 have been tested numerically in order to assess the quality of the rotation computation method during very large deformations. For these simulations, the strain rate was $\dot{\gamma}=\sqrt{3} \mathrm{~s}^{-1}$. The rate of rotation of the local axes as a function of the shear strain was particularly focused on. The three methods presented in Section 4 are compared in Fig. 15. For the Mandel spin approach, the rate of rotation is computed during FE simulations with one finite element. While, for the two other methods, this rotation rate can be computed analytically as a function of the shear strain. For the CSLVG method, the rate of rotation is constant:

$\dot{\varphi}=\dot{\gamma} / 2=\sqrt{3} / 2 \mathrm{rad} / s=49.62^{\circ} / \mathrm{s}$

For the co-rotational approach, the rotation of the local frame is the half of the rotation of the material frame, which is directly related to the shear strain:

$$
\varphi=\frac{\varphi_{1}}{2}=\frac{\operatorname{atan}(\gamma)}{2} .
$$

Then, the rate of rotation is obtained:

$\dot{\varphi}=\frac{\dot{\gamma}}{2\left(1+\gamma^{2}\right)}$.

The results presented in Fig. 15 are in good agreement with similar numerical results of Peeters et al. (2001). The decrease of the rate of rotation with the Mandel spin approach is due to the evolution of the texture towards more stable components with respect to shear straining. It can be deduced that the rate of rotation computed with the Mandel spin approach is largely depending on the texture of the material (the initial texture and its evolution), through stable or non-stable components of texture with regard to the imposed strain path (Bacroix and $\mathrm{Hu}$, 1995).

Besides, the rate of rotation of the local frame computed with the CSLVG or the co-rotational approaches are independent of the material behaviour. In the current case, the results with the co-rotational approach are close to the reference results of the Mandel spin approach where the texture update is taken into account. But the decrease observed with the co-rotational approach is purely geometric. For other materials, the agreement could be worse. For instance, if a material with stable texture components with respect to shearing was tested, the CSLVG and the co-rotational approaches would yield to inaccurate results (large overestimation of the rate of rotation for small strains). 
For the shearing process investigated in the present paper, according to Fig. 15, it appears that the CSLVG approach (Jaumann type approach) is only valid for small strains $(\gamma<0.3)$ where the Mandel spin represents 93.3\% of the Jaumann type spin. For larger strains, it hugely overestimates the rate of rotation (e.g. for $\gamma=0.3$, the Mandel spin represents only $76 \%$ of the Jaumann type spin). These computations performed in the context of finite element simulation where numerical approximations occur, discretization, etc., remain in good agreement with Peeters et al. (2001).

Fig. 15: Evolution of the rate of rotation of the local frame for the three investigated methods. The shear strain $\gamma$ was ranging from 0 to 3.5 with a constant strain rate $\dot{\gamma}$ of $\sqrt{3} \mathrm{~s}^{-1}$

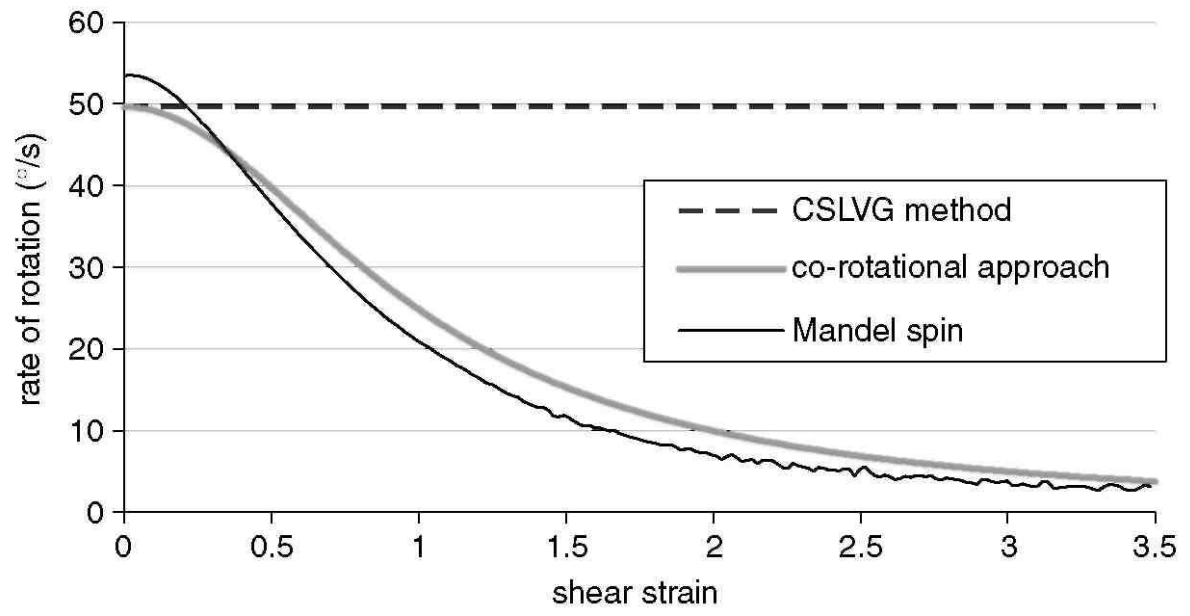

\section{CONCLUSION}

The purpose of the present work is the investigation of different ways to determine a local frame being the best compromise to express the rotation of a constant yield locus (when no updating of the yield locus is taken into account), even if large strains occur. Three distinct approaches are presented and tested:

- A kinematics based approach (Jaumann type) named Constant Symmetric Local Velocity Gradient (CSLVG),

- A co-rotational approach founded on geometrical considerations,

- The Mandel spin approach computed from crystal orientation evolution, which corresponds to a physical approach. In this case, four different methods where adopted in order to compute the Mandel spin $\mathrm{W}^{\text {lattice }}$.

The analyses carried out here are performed taking into account or not the crystallo-graphic texture evolution using the Full Constraints Taylor's model. For the cold rolled IF mild steel used in this work, the predicted texture evolution is compared to the experimental one (Figs. 13 and 14) in order to assess the capability of the adopted micro-mechanical model. Favourable agreement to the experimental measurements is observed.

The sensitivity of the experimental and numerical results to the boundaries conditions is analysed. During the simple shear test, full-field measurement technique is used in order to compute the kinematics field on the entire surface of the gauge area. The rotation of the material frame in the centre of the specimen is measured and compared to the imposed macroscopic amount of shear strain. In the same way, FE computations are carried out using one or a huge number of elements. The rotation of the material and local frames in the centre of the specimen are compared. Very small $(<2 \%)$ sensitivity to the boundaries conditions is observed.

The sensitivity of the results to the constitutive laws is also taken under consideration through the yield locus description (i.e. the quadratic Hill, 1948, the local yield locus description without (Minty) or with texture update $($ Evol)). Very small variation $(<2 \%)$ of the predicted rotation of the material and the local frames is observed when the yield surface description changes. 
The main conclusions that can be drawn when comparing the different approaches used for the computation of the FE local axes evolution are:

(a) For small shear strain, the three methods yield to almost similar results. The CSLVG method is less accurate. Contrarily, the Mandel spin approach should be regarded as a reference due to its physical roots but it imposes the use of a texture based constitutive model with computation of the texture evolution.

(b) For large shear strains, the texture evolution has an impact on the evolution of the local frame. More physical result is obtained with the Mandel spin (i.e. the rotation rate tends to zero, which is not the case when Jaumann type spin is adopted). The Mandel spin approach is therefore preferred. According to Fig. 15, it appears that the co-rotational approach gives accurate results (compared to the Mandel spin approach) for shear strain up to 3.5. But it has the important drawback of being independent of the material behaviour.

(c) The results obtained in the present work with the Mandel spin approach are in agreement with the observations reported in Peeters et al. (2001) and Bacroix et al. (1994) for IF mild steel sheet subjected to simple shear. The rotation axis and the rotation angle are both consistent. Compared to the previous research work, the present investigations based on macroscopic FEM solutions, are achieved with numerical models (micro-macro constitutive law, texture evolution computation and the three methods for the computation of the local axes) fully implemented in a finite element code.

(d) The different methods proposed for the computation of the Mandel spin yield to very close results. The variation from the average value of the predicted rotation of the local frame is less than $3 \%$. This is explained by the fact that the texture update is performed each increment of plastic strain. Sensitivity of the rotation of the local frame to the computational method can be observed when the texture update is performed at large interval of plastic strain $(\approx 10-20 \%)$.

\section{Acknowledgements}

As Research Director of the National Fund for Scientific Research, A.M. Habraken thanks this Belgian research fund for its support. The authors acknowledge the Belgian Federal Science Policy Office for its financial support and its efficient cooperation impulsion between partners of IAP5/08. Professor Paul Van Houtte is thanked for his great contribution in providing texture measurements, texture analysis software and valuable discussions.

\section{References}

Bacroix, B., Hu, Z., 1995. Texture evolution during strain path changes in low carbon steel sheets. Metall. Trans.A 26A, 601-613.

Bacroix, B., Genevois, P., Teodosiu, C, 1994. Plastic anisotropy in low carbon steels subjected to simple shear with strain path changes. Eur J. Mech. A/Solids 13, 661-675.

Barlat, F., Lege, D.J., Brem, J.C., 1991. A six-component yield function for anisotropic materials. Int. J. Plast. 7, 693-712.

Belytschko, T., Bindeman, L.P., 1991. Assumed strain stabilization of the 4-node quadrilateral with 1-point quadrature for nonlinear problems. Comput. Methods Appl. Mech. Engrg. 88, 311-340.

Bouvier, S., Haddadi, H., Levée, P., Teodosiu, C, 2006. Simple shear tests: experimental techniques and characterization of the plastic anisotropy of rolled sheets at large strains. J. Mater. Process. Technol. 172, 96-103.

Casotto, S., Pascon, F., Habraken, A.M., Bruschi, S., 2005. Thermo-mechanical-metallurgical model to predict geometrical distortions of rings during cooling phase after ring rolling operations. Int. J. Machine Tools Manufact. 45, 657-664.

Castagne, S., Remy, M., Habraken, A.M., 2003. Development of a mesoscopic cell modeling the damage process in steel at elevated temperature. In: Engrg. Mat., vols. 223-236. Trans. Tech. Publications, Switzerland, pp.145-150.

Castagne, S., Pascon, F., Bles, G., Habraken, A.M., 2004. Developments in finite element simulations of continuous casting. J. Phys. IV 120, 447-455.

Cescotto, S., 1984. Finite deformation of solids. In: Hartley, P., Pillinger, I., Sturgess, C. (Eds.), Numerical Modelling of Material Deformation Processes. Springer-Verlag, pp. 20-67. 
Cescotto, S., Charlier, R., 1993. Frictional contact finite element based on mixed variational principles. Int. J. Numer. Methods Engrg. 36 , $1681-1701$.

Dafalias, Y.F., 1983. Corotational rates for kinematic hardening at large plastic deformation. J. Appl. Mech. ASME 50, $561-565$.

Dafalias, Y.F., 1985. The plastic spin. J. Appl. Mech. ASME 52, 865-871.

Dafalias, Y.F., 1998. Plastic spin: necessity or redundancy? Int. J. Plasticity 14, 909-931.

Dai, J.S., 2006. An historical review of the theoretical development of rigid body displacements from Rodrigues parameters to the finite twist. Mech. Mach. Theory 41, 41-52.

Dawson, P.R., Boyce, D.E., Hale, R., Durkot, J.P., 2005. An isoparametric piecewise representation of the anisotropic strength of polycrystalline solids. Int. J. Plasticity 21, 251-283.

Delannay, L., Jacques, P. J., Kalidindi, S.R., 2006. Finite element modeling of crystal plasticity with grains shaped as truncated octahedrons Int. J. Plasticity 22, 1879-1898.

Drean, M., Habraken, A.M., Bouchair, A., Muzeau, J.P., 2002. Swaged bolts: modelling of the installation process and numerical analysis of the mechanical behaviour. Comput. Struct. 80, 2361-2373.

Duchêne, L., 2003. FEM study of metal sheets with a texture based, local description of the yield locus. Ph.D. Thesis, Ulg, Liège, Belgium.

Duchêne, L., Habraken, A.M., 2005. Analysis of the sensitivity of FEM predictions to numerical parameters in deep drawing simulations Eur. J. Mech. A/Solids 24, 614-629.

Duchêne, L., Godinas, A., Cescotto, S., Habraken, A.M., 2002. Texture evolution during deep-drawing processes. J. Mater. Process Technol., 110-118.

Duchêne, L., de Montleau, P., El Houdaigui, F., Bouvier, S., Habraken, A.M., 2005. Analysis of texture evolution and hardening behavior during deep drawing with an improved mixed type FEM element. In: Smith, L.M., Pourboghrat, F., Yoon, J-W., Stoughton, T.B. (Eds.), Proc. Int. Conf. NUMISHEET 2005, Melville, New York, vol. 1, pp. 409-414.

Duchêne, L., El Houdaigui, F., Habraken, A.M., 2007. Length changes and texture prediction during free end torsion test of copper bars with fem and remeshing techniques. Int. J. Plasticity 23, 1417-1438.

Flores, P., 2005. Development of experimental equipment and identification procedures for sheet metal constitutive laws. Ph.D. Thesis University of Liege, Belgium.

Flores, P., Rondia, E., Habraken, A.M., 2005. Development of experimental equipment for the identification of constitutive laws. Int. J. Form. Process, special issue, 117-137.

Gom, 2001. ARAMIS, Deformation measurement using the grating method, GOM mbH, Braunschweig.

Habraken, A.M., Bourdouxhe, M., 1992. Coupled thermo-mechanical-metallurgical analysis during the cooling of steel pieces. Eur. J. Mech. A/Solids 11 (3), 381-402

Habraken, A.M., Cescotto, S., 1990. An automatic remeshing technique for finite element simulation of forging processes. Int. J. Numer. Methods Engrg. 30, 1503-1525.

Habraken, A.M., Cescotto, S., 1998. Contact between deformable solids, the fully coupled approach. Math. Comput. Modell. 28 (4-8), 153169.

Habraken, A.M., Duchêne, L., 2004. Anisotropic elasto-plastic finite element analysis using a stress-strain interpolation method based on a polycrystalline model. Int. J. Plasticity 20, 1525-1560.

Habraken, A.M., Charles, J.F., Wegria, J., Cescotto, S., 1998. Dynamic recrystallization during zinc rolling. Int. J. Form. Process. 1, 1-20.

Hill, R., 1948. A theory of the yielding and plastic flow of anisotropic metals. Proc. Roy. Soc. London A 193, $281-297$.

Hughes, T.J.R., 1983. Theoretical foundation for large-scale computations of nonlinear material behaviour. In: Nemat-Nasser, S., Asaro, R.J., Hegemier, G.A. (Eds.), Proc. Workshop on Theor. Foundation for Large- Scale Computations of Nonlinear Material Behaviour, Evanston, Illinois. Martinus Nijhoff Publisher, Dordrecht, pp. 29-63.

Johnson, K.I., Nguyen, B.N., Davies, R.W., Grant, G.J., Khaleel, M.A., 2004. A numerical process control method for circular-tube hydroforming prediction. Int. J. Plasticity 20, 1111-1137. 
Published in: International Journal of Plasticity (2008), iss. 24, pp 397-427

Status: Postprint (Author's version)

Kim, D., Barlat, F., Bouvier, S., Rabahallah, M., Balan, T., Chung, K., 2007. Non-quadratic anisotropic potentials based on linear transformation of plastic strain rate. Int. J. Plasticity 23, 1380-1399.

Li, K.P., Cescotto, S., 1997. An 8-node brick element with mixed formulation for large deformation analyses. Comput. Methods Appl. Mech. Engrg. 141, 157-204

Li, K.P., Habraken, A.M., Bruneel, H., 1995. Simulation of square-cup deep-drawing with different finite elements. J. Mater. Process. Technol. 50, 81-91.

Mandel, J., 1982. Définition d'un repère privilégié pour l'étude des transformations anélastiques du polycrystal. J. Méc. Théo. Appli. 1, 7.

Munhoven, S., Habraken, A.M., 1995. Application of an anisotropic yield locus based on texture to a deep drawing simulation. In: Shen, S.E., Dawson, P.R. (Eds.), Simulation of Materials Processing: Theory, Methods and Applications, NUMIFORM 95. Balkema, Rotterdam.

Peeters, B., Hoferlin, E., Van Houtte, P., Aernoudt, E., 2001. Assessment of crystal plasticity based calculation of the lattice spin of polycrystalline metals for FE implementation. Int. J. Plasticity 17, 819-836.

Ponthot, J.P., 2002. Unified stress update algorithms for the numerical simulation of large deformation elasto-plastic and elasto-viscoplatic processes. Int. J. Plasticity 18, 91-126.

Toth, L.S., Jonas, J.J., Gilormini, P., Bacroix, B., 1990. Length changes during free end torsion: a rate sensitive analysis. Int. J. Plasticity 6, 83-108.

Van Der Giessen, E., 1991. Micromechanical and thermodynamic aspects of the plastic spin. Int. J. Plasticity 7, 365-386.

Van Houtte, P., 1994. The 'MTM-FHM' software system, version 2, User's manual, KUL, Leuven.

Van Houtte, P., 2001. Fast computation of average Taylor factors and Mandel spins for all possible strain modes. Int. J. Plasticity 17, 807818.

Van Houtte P., Rabet L., 1997. Generalisation of the relaxed constraints models for the prediction of deformation textures. La revue de Métallurgie - CIT/Science et Génie des Matériaux, pp. 1483-1494.

Van Houtte, P., Van Bael, A., 2004. Convex plastic potentials of fourth and sixth rank for anisotropic materials. Int. J. Plasticity 20, 15051524.

Wang, J., Wagoner, R.H., 2004. A new hexahedral solid element for 3D FEM simulation of sheet metal forming. In: Ghosh, S. et al. (Eds.), AIP Conf. Proc, Proc. NUMIFORM, vol. 712, pp. 2181-2186.

Wu, H-C, 2007. On stress rate and plasticity constitutive equations referred to a body-fixed coordinate system. Int. J. Plasticity 23, 14861511.

Yoon, J.W., Barlat, F., Dick, R.E., Chung, K., Kang, T.J., 2004. Plane stress yield function for aluminum alloy sheets-part II: FE formulation and its implementation. Int. J. Plasticity 20, 495-522.

Yoon, J.W., Barlat, F., Gracio, J.J., Rauch, E., 2005. Anisotropic strain hardening behavior in simple shear for cube textured aluminum alloy sheets. Int. J. Plasticity 21, 2426-2447.

Yoon, J.W., Barlat, F., Dick, R.E., Karabin, M.E., 2006. Prediction of six or eight ears in a drawn cup based on a new anisotropic function. Int. J. Plasticity 22, 174-193.

Zhu, Y.Y., Cescotto, S., 1994. Transient thermal and thermomechanical analysis by FEM. Comput. Struct. 53, 275-304.

Zhu, Y.Y., Cescotto, S., 1995a. Unified and mixed formulation of the 4-node quadrilateral elements by assumed strain method: application to thermomechanical problems. Int. J. Numer. Methods Engrg. 38, 685-716.

Zhu, Y.Y., Cescotto, S., 1995b. A fully coupled elasto-visco-plastic damage theory for anisotropic materials. Int. J. Solids Struct. 32 (11), 1607-1641. 